\title{
The Structure of Autocovariance Matrix of Discrete Time Subfractional Brownian Motion
}

\author{
Guo Jiang \\ School of Mathematics and Statistics, Hubei Normal University, Huangshi 435002, China \\ Correspondence should be addressed to Guo Jiang; gjiang@hbnu.edu.cn
}

Received 7 October 2017; Revised 11 December 2017; Accepted 19 December 2017; Published 20 May 2018

Academic Editor: Marcello Vasta

Copyright (C) 2018 Guo Jiang. This is an open access article distributed under the Creative Commons Attribution License, which permits unrestricted use, distribution, and reproduction in any medium, provided the original work is properly cited.

This article explores the structure of autocovariance matrix of discrete time subfractional Brownian motion and obtains an approximation theorem and a structure theorem to the autocovariance matrix of this stochastic process. Moreover, we give an expression to the unique time varying eigenvalue of the autocovariance matrix in asymptotic means and prove that the increments of subfractional Brownian motion are asymptotic stationary processes. At last, we illustrate these results with numerical experiments and give some probable applications in finite impulse response filter.

\section{Introduction}

Fractional Brownian motion (FBm) $\left\{B_{t}^{H}, t \geq 0\right\}$ is a continuous centered Gaussian process starting from zero and with covariance

$$
R_{H}(s, t)=E\left[B_{t}^{H} B_{s}^{H}\right]=\frac{V_{H}}{2}\left(t^{2 H}+s^{2 H}-|t-s|^{2 H}\right),
$$

where $H \in(0,1), V_{H}=1 / \Gamma(2 H+1)|\sin H \pi|$. When $H=$ $1 / 2, \mathrm{fBm}$ is a standard Brownian motion (see $[1,2]$ and their references).

Subfractional Brownian motion (SfBm) $\left\{S_{t}^{H}, t \geq 0\right\}$ had been introduced by Bojdecki et al. [3]; it is also a continuous Gaussian process starting from zero and with covariance

$$
\begin{aligned}
& C_{H}(s, t)=E\left[S_{t}^{H} S_{s}^{H}\right] \\
& \quad=V_{H}\left(t^{2 H}+s^{2 H}-\frac{1}{2}\left((t+s)^{2 H}+|t-s|^{2 H}\right)\right) .
\end{aligned}
$$

When $H \in(1 / 2,1)$, it arises from occupation time fluctuation of branching particle systems (see [3-5]). Moreover, sfBm has some properties analogous to those of $\mathrm{fBm}$, such as selfsimilarity, long-rang dependence, and Hölder continuity (for details we refer to [6-9]). But sfBm has nonstationary increments, its increments over nonoverlapping intervals are more weakly correlated, and the covariance decays polynomially at a higher rate in comparison with $\mathrm{fBm}$; thereby it is called $\mathrm{sfBm}$ in [3].

It is well known that $\mathrm{fBm}$ has been successfully applied in the engineering fields like characterization of texture in bone radiographs, image processing and segmentation, terrain surface modeling, medical image analysis, and network traffic analysis. However, in contrast to the extensive applications on $\mathrm{fBm}$, there have been lesser systematic investigations on $\mathrm{sfBm}$. The main reason for this is the complexity of dependence structures for the process which has not stationary increments. Therefore, it seems meaningful to study the structure of autocovariance matrix of discrete time sfBm.

In recent years, Tudor [7] studied some new properties of $\mathrm{sfBm}$ such as strong variation, renormalized power variation, the Dirichlet property, and short and long memory properties. Moreover, in [8, 9], Tudor characterized the domain of the Wiener integral with respect to $\mathrm{sfBm}$. Yan et al. [10] also defined stochastic integral with respect to $\mathrm{sfBm}$ with $H \in(1 / 2,1)$ and obtained Itô and Tanaka formulas. Shen and Chen [6] defined the extended divergence integral with respect to sfBm with $H \in(0,1 / 2)$ and established the corresponding versions of Itô and Tanaka formulas. Shen and Yan [11] and Rao [12] give the efficient estimation for the drift term parameter driven by sfBm. Shen and Yan [13] obtain an approximation theorem for sfBm using martingale differences. Liu et al. $[14,15]$ study the estimators for the selfsimilarity of $\mathrm{sfBm}$. 
On the other hand, covariance matrix is a very important feature of stochastic processes. These studies to covariance matrix are always a significant and interesting topic in probability and statistics. There exist large amounts of literatures about theories and applications of covariance matrix (see [16-23], etc.). In fact, one usually works with discrete time stochastic processes for practical application purposes (e.g., [17, 24-26]). In particular, Ayache et al. [24] presented the explicit covariance formula of multifractional Brownian motion and briefly reported some applications in the synthesis problem and the long-term structure. In [17], Gupta and Joshi studied the structure of covariance matrix of discrete time $\mathrm{fBm}$ and gave the application in $M$-band wavelet system. Yet the corresponding researches are still blank space for the $\mathrm{sfBm}$ which has nonstationary increments.

Inspired by these, we explore the structure of autocovariance matrix of discrete time $\mathrm{sfBm}(\mathrm{dtsfBm})$, and then we obtain an approximation theorem and a structure theorem to the autocovariance matrix of dtsfBm. At the same time, we give an expression to the unique time varying eigenvalue of the autocovariance matrix in asymptotically means and prove that the increments of sfBm are asymptotically stationary. At last, we illustrate these results with numerical experiments and give some probable application in finite impulse response filter. These researches fill the gap between the theories and applications of $\mathrm{sfBm}$.

This paper is organized as follows: In Section 2, we present some preliminaries about sfBm and matrix theory. In Section 3, we give the approximation to the autocovariance matrix of dtsfBm, testify the unique time varying eigenvalue in asymptotic sense, and prove that these increments of dtsfBm are asymptotically stationary. Then, we establish the structure theorem (Theorem 17) to the autocovariance matrix of dtsfBm. In Section 4, we illustrate those results obtained in Section 3 by numerical experiments and give some probable applications. In Section 5, we come to the conclusion of the paper.

\section{Preliminaries}

2.1. Discrete Time Subfractional Brownian Motion. In fact, we often observe the discrete time signals. SfBm could be used to model some random conditions. This article exactly concerns the statistical properties of dtsfBm and presents some results on the structure of its autocovariance matrix. DtsfBm is defined as

$$
S_{n}^{H}=S_{n T_{s}}^{H}
$$

where $n \in \mathbb{N}$ and $T_{s}$ is the sampling period. For convenience, one usually lets $T_{s}=1$. Since sfBm is self-similar, we have $S_{n}^{H}=n^{H} S_{1}^{H}$. Moreover, the mean value, variance, and autocovariance function of $\mathrm{dtsfBm}$ are given as follows:

$$
\begin{aligned}
& E S_{n}^{H}=0, \\
& \operatorname{Var} S_{n}^{H}=\operatorname{Var}\left(n^{H} S_{1}^{H}\right)=n^{2 H} \operatorname{Var}\left(S_{1}^{H}\right)=n^{2 H} V_{H},
\end{aligned}
$$

$$
\begin{gathered}
C_{H}\left(n_{1}, n_{2}\right)=E\left[S_{n_{1}}^{H} S_{n_{2}}^{H}\right]=V_{H}\left(n_{1}{ }^{2 H}+n_{2}{ }^{2 H}\right. \\
\left.-\frac{1}{2}\left(\left(n_{1}+n_{2}\right)^{2 H}+\left|n_{1}-n_{2}\right|^{2 H}\right)\right) .
\end{gathered}
$$
that is,

Let $Y_{n, m}$ be an $m$-dimensions random vector of dtsfBm;

$$
Y_{n, m}=\left(S_{n}^{H}, S_{n+1}^{H}, S_{n+2}^{H}, \ldots, S_{n+m-1}^{H}\right)^{T}
$$

In the following, we mainly study the autocovariance of the vector $Y_{n, m}$ as follows:

$$
\begin{aligned}
\mathbf{K}(n, m) & =E\left[Y_{n, m} Y_{n, m}^{T}\right]=\left(E\left[S_{n+i-1}^{H} S_{n+j-1}^{H}\right]\right)_{m \times m}^{(n)} \\
& =\left(k_{i j}\right)_{m \times m}^{(n)},
\end{aligned}
$$

where

$$
\begin{aligned}
k_{i j} & =V_{H} \cdot\left((n+i-1)^{2 H}+(n+j-1)^{2 H}\right. \\
& \left.-\frac{1}{2}\left((2 n+i+j-2)^{2 H}+|i+j-2|^{2 H}\right)\right),
\end{aligned}
$$

and $i, j=1,2, \ldots, m$.

Obviously, sfBm is nonstationary and the autocovariance matrix is a function in $n$.

2.2. Some Results of the Matrix Theory. In this section, we recall some useful results of the matrix theory (for details see also [27]).

Let $\mathbf{A} \in \mathbb{C}^{n \times n}$ be a matrix, $\lambda_{1}, \lambda_{2}, \ldots, \lambda_{s}$ are the distinct eigenvalues of $\mathbf{A}$, and

$$
p(\lambda)=\left(\lambda-\lambda_{1}\right)^{m_{1}}\left(\lambda-\lambda_{2}\right)^{m_{2}} \cdots\left(\lambda-\lambda_{s}\right)^{m_{s}}
$$

is the minimal polynomial of $\mathbf{A}$ with degree $m^{*}=m_{1}+m_{2}+$ $\cdots+m_{s}$.

Theorem 1 (see [27, P 314]). If a matrix $\mathbf{A} \in \mathbb{C}^{n \times n}$, a given function $f(\lambda)$ is defined on the spectrum $\sigma(\mathbf{A})=\left\{\lambda_{1}, \lambda_{2}, \ldots\right.$, $\lambda_{s}$ \} of $\mathbf{A}$, and $f_{k, j}$ denote the value of the $j$ th derivative of $f(\lambda)$ at the eigenvalue $\lambda_{k}\left(k=1,2, \ldots, s ; j=0,1, \ldots, m_{k}-1\right)$, then there exist component matrices $\mathbf{Z}_{k j}$ which are independent of $f(\lambda)$ and

$$
f(\mathbf{A})=\sum_{k=1}^{s} \sum_{j=0}^{m_{k}-1} f_{k, j} \mathbf{Z}_{k j}
$$

Moreover, the matrices $\mathbf{Z}_{k j}$ are linearly independent members of $\mathbb{C}^{n \times n}$ and commutative with $\mathbf{A}$ and with each other.

In particular, where $z \notin \sigma(\mathbf{A})$, the resolvent $\mathbf{R}_{z}=(z \mathbf{I}-\mathbf{A})^{-1}$ of $\mathbf{A}$ can be expressed as

$$
\mathbf{R}_{z}=(z \mathbf{I}-\mathbf{A})^{-1}=\sum_{k=1}^{s} \sum_{j=0}^{m_{k}-1} \frac{j !}{\left(z-\lambda_{k}\right)^{j+1}} \mathbf{Z}_{k j} .
$$


Theorem 2 (see [27, $\mathrm{P}$ 315]). The components $\mathbf{Z}_{k j}(k=$ $\left.1,2, \ldots, s ; j=0,1, \ldots, m_{k}-1\right)$ of the matrix $\mathbf{A} \in \mathbb{C}^{n \times n}$ satisfy the following conditions:

$$
\begin{aligned}
\sum_{k=1}^{s} \mathbf{Z}_{k 0} & =\mathbf{I}, \\
\mathbf{Z}_{k j} \mathbf{Z}_{l q} & =0, \quad \text { if } k \neq l, \\
\mathbf{Z}_{k j}^{2} & =\mathbf{Z}_{k j}, \quad \text { iff } j=0, \\
\mathbf{Z}_{k 0} \mathbf{Z}_{k j} & =\mathbf{Z}_{k j}, \quad j=0,1, \ldots, m_{k}-1,
\end{aligned}
$$

where, for $k=1,2, \ldots, s$ and $m_{1}=m_{2}=\cdots=m_{s}=1$,

$$
\mathbf{Z}_{k 0}=\frac{\prod_{l=1, l \neq k}^{s}\left(\mathbf{A}-\lambda_{l} \mathbf{I}\right)}{\prod_{l=1, l \neq k}^{s}\left(\lambda_{k}-\lambda_{l}\right)} .
$$

Theorem 3 (see [27, P 319]). For $k=1,2, \ldots, s$ and $j=$ $0,1, \ldots, m_{k}-1$,

$$
\mathbf{Z}_{k j}=\frac{1}{j !}\left(\mathbf{A}-\lambda_{k} \mathbf{I}\right)^{j} \mathbf{Z}_{k 0} .
$$

We now present some relevant results on analytic perturbation of linear operator.

Lemma 4 (see [27, P 392]). Let $\mathbf{A} \in \mathbb{C}^{n \times n}$ be an unperturbed matrix with eigenvalues $\lambda_{1}, \lambda_{2}, \ldots, \lambda_{n} . \mathbf{A}(\varepsilon)$ is an $n \times n$ matrix whose elements are analytic functions of complex number $\varepsilon$ in a neighborhood of $\varepsilon=0$ such that

$$
\mathbf{A}(\varepsilon)=\mathbf{A}+\varepsilon \mathbf{A}^{(1)}+\varepsilon^{2} \mathbf{A}^{(2)}+\cdots,
$$

the eigenvalues $\lambda_{1}(\varepsilon), \lambda_{2}(\varepsilon), \ldots, \lambda_{n}(\varepsilon)$ of $\mathbf{A}(\varepsilon)$ depend continuously on $\varepsilon$, and $\lambda_{k}(\varepsilon) \rightarrow \lambda_{k}$ as $|\varepsilon| \rightarrow 0$ for $k=1,2, \ldots, n$, where $\mathbf{A}^{(1)}, \mathbf{A}^{(2)}, \ldots \in \mathbb{C}^{n \times n}$ and the superscripts do not denote derivatives in this paper; the following results are established:

(i) if $\lambda_{k}$ is an unrepeated eigenvalue of $\mathbf{A}$, then $\lambda_{k}(\varepsilon)$ is analytic in a neighborhood of $\varepsilon=0$;

(ii) if $\lambda_{k}$ has algebraic multiplicity $m$ and $\lambda_{k p}(\varepsilon) \rightarrow \lambda_{k}$ for $p=1,2, \ldots, m$, then $\lambda_{k p}(\varepsilon)$ is an analytic function of $\varepsilon^{1 / l}$ in a neighborhood of $\varepsilon=0$, where $l \leq m$ and $\varepsilon^{1 / l}$ is one of the $l$ branches of the function $\varepsilon^{1 / l}$. Thus, $\lambda_{k p}(\varepsilon)$ has a power series expansion in $\varepsilon^{1 / l}$.

Remark 5. The perturbed eigenvalues remain real for a Hermitian matrix; the component matrices of $\mathbf{A}(\varepsilon)$ associated with perturbed eigenvalues will be Hermitian and analytic in $\varepsilon$, whatever the multiplicity of the unperturbed eigenvalues may be. The eigenvectors of $\mathbf{A}(\varepsilon)$ are analytic and orthonormal throughout a neighborhood of $\varepsilon=0$.

Lemma 6 (see [27, P 396]). Let $\mathbf{A}(\varepsilon)$ be a matrix that is analytic in $\varepsilon$ on a neighborhood of $\varepsilon=0$, and $\mathbf{A}(0)=\mathbf{A}$. Let $\lambda_{k}$ be an unrepeated eigenvalue of $\mathbf{A}$ with index one; then, for sufficiently small $|\varepsilon|$, there is an eigenvalue $\lambda_{k}(\varepsilon)$ of $\mathbf{A}(\varepsilon)$ such that

$$
\lambda_{k}(\varepsilon)=\lambda_{k}+\varepsilon \lambda_{k}^{(1)}+\varepsilon^{2} \lambda_{k}^{(2)}+\cdots
$$

Moreover, there are right and left eigenvectors $X_{k}(\varepsilon)$ and $Y_{k}(\varepsilon)$, respectively, associated with $\lambda_{k}(\varepsilon)$ for which

$$
\begin{aligned}
Y_{k}^{T}(\varepsilon) X_{k}(\varepsilon) & =1, \\
X_{k}(\varepsilon) & =X_{k}+\varepsilon X_{k}^{(1)}+\varepsilon^{2} X_{k}^{(2)}+\cdots, \\
Y_{k}(\varepsilon) & =Y_{k}+\varepsilon Y_{k}^{(1)}+\varepsilon^{2} Y_{k}^{(2)}+\cdots
\end{aligned}
$$

Remark 7 (see [27, P 399]). (I) The first-order perturbation coefficients are as follows:

$$
\begin{gathered}
\lambda_{k}^{(1)}=Y_{k}^{T} \mathbf{A}^{(1)} X_{k}, \\
X_{k}^{(1)}=\mathbf{E}_{k} \mathbf{A}^{(1)} X_{k},
\end{gathered}
$$

where the matrix $\mathbf{E}_{k}$ is defined as

$$
\begin{aligned}
\mathbf{E}_{k} & =\lim _{z \rightarrow \lambda_{k}}(z \mathbf{I}-\mathbf{A})^{-1}\left(\mathbf{I}-\mathbf{Z}_{k 0}\right) \\
& =\sum_{l=1, l \neq k}^{s} \sum_{r=0}^{m_{l}-1} \frac{r !}{\left(\lambda_{k}-\lambda_{l}\right)^{r+1}} \mathbf{Z}_{l r} .
\end{aligned}
$$

(II) In particular, if the perturbation of $\mathbf{A}$ is linear in $\varepsilon$, that is, $\mathbf{A}(\varepsilon)=\mathbf{A}+\varepsilon \mathbf{A}^{(1)}$, then the perturbation coefficients for all orders are given by

$$
\begin{aligned}
& \lambda_{k}^{(r)}=Y_{k}^{T} \mathbf{A}^{(1)} X_{k}^{(r-1)}, \quad r=1,2, \ldots \\
& X_{k}^{(r)}=\mathbf{E}_{k}\left(\mathbf{A}^{(1)} X_{k}^{(r-1)}-\sum_{q=1}^{r-1} \lambda_{k}^{(q)} X_{k}^{(r-q)}\right),
\end{aligned}
$$

$$
r=1,2, \ldots
$$

Lemma 8 (see [27, P 402]). Let $\mathbf{A}(\varepsilon)$ be a matrix that is analytic in $\varepsilon$ on a neighborhood of $\varepsilon=0$, and $\mathbf{A}(0)=\mathbf{A}$. $\lambda_{k}$ is an eigenvalue of $\mathbf{A}$ with index one and multiplicity $m$; then the eigenvalue $\lambda_{k}$ splits into $\lambda_{k 1}(\varepsilon), \lambda_{k 2}(\varepsilon), \ldots, \lambda_{k m}(\varepsilon)$ for sufficiently small $|\varepsilon|$. Let $\lambda_{k p}(\varepsilon)(p=1,2, \ldots, m)$ be the eigenvalues of $\mathbf{A}(\varepsilon)$ and $\lambda_{k p}(0)=\lambda_{k}(p=1,2, \ldots, m)$. Then there is a number $a_{k p}$ and a positive integer $l \leq m$ such that

$$
\lambda_{k p}(\varepsilon)=\lambda_{k}+a_{k p} \varepsilon+o\left(|\varepsilon|^{1+1 / l}\right), \quad|\varepsilon| \longrightarrow 0,
$$

where $a_{k p}$ is an eigenvalue of $\mathbf{Z}_{k 0} \mathbf{A}^{(1)} \mathbf{Z}_{k 0}$, and

$$
\mathbf{Z}_{k 0}=\frac{1}{2 \pi \mathbf{i}} \int_{L_{k}} \mathbf{R}_{z} \mathrm{~d} z,
$$

with $L_{k}$ being a simple closed contour that encircles $\lambda_{k}, \lambda_{k 1}(\varepsilon)$, $\lambda_{k 2}(\varepsilon), \ldots, \lambda_{k m}(\varepsilon)$ and no other eigenvalues of $\mathbf{A}$ or $\mathbf{A}(\varepsilon)$.

Moreover, there is at least one eigenvector $X_{k p}(\varepsilon)$ which corresponds to each $\lambda_{k p}(\varepsilon)$ such that

$$
X_{k p}(\varepsilon)=X_{k}+\varepsilon^{1 / l} X_{k, 1}+\varepsilon^{2 / l} X_{k, 2}+\cdots, \quad|\varepsilon| \longrightarrow 0,
$$


with $X_{k}, X_{k, 1}, \ldots, X_{k, l-1} \in \operatorname{Ker}\left(\mathbf{A}-\lambda_{k} \mathbf{I}\right)$ and $a_{k p} X_{k}=$ $\mathbf{Z}_{k 0} \mathbf{A}^{(1)} \mathbf{Z}_{k 0} X_{k}$.

Lemma 9 (see [27, P 403]). If $a_{k p}$ is an eigenvalue of $\mathbf{Z}_{k 0} \mathbf{A}^{(1)} \mathbf{Z}_{k 0}$ with index one, then there exists $b_{k p}$ such that

$$
\lambda_{k p}(\varepsilon)=\lambda_{k}+a_{k p} \varepsilon+b_{k p} \varepsilon^{2}+o\left(|\varepsilon|^{2+1 / l}\right)
$$

where $b_{k p}$ is an eigenvalue of $\mathbf{Z}_{k 0}\left(\mathbf{A}^{(1)} \mathbf{E}_{k} \mathbf{A}^{(1)}+\mathbf{A}^{(2)}\right) \mathbf{Z}_{k 0}$ with right eigenvector $X_{k}$.

\section{Main Results}

In this section, we study the autocovariance matrix of dtsfbm and give the approximation theorem of the matrix. At the same time, we give an expression to the unique time varying eigenvalue of the autocovariance matrix in asymptotic means (i.e., for large enough $n \geq n_{\min }$ ). Though the increments of $\mathrm{sfBm}$ are nonstationary, we show that the increments of dtsfBm are asymptotically stationary and obtain a structure theorem about the autocovariance matrix.

Theorem 10 (approximation theorem). Let $Y_{n, m}$ be a random vector of length $m$ of dtsfBm (see (7)) and let $\mathbf{K}(n, m)$ be $m \times m$ autocovariance matrix of the vector $Y_{n, m}$; then $\mathbf{K}(n, m)$ can be approximated as $\widehat{\mathbf{K}}(n, m)$ for large enough $n \geq n_{\min }$, and

$$
\widehat{\mathbf{K}}(n, m)=\left(\widehat{k}_{i j}\right)_{m \times m}^{(n)}=c_{0} n^{2 H} V_{H} \cdot\left(\mathbf{A}+\varepsilon_{1} \mathbf{B}+\varepsilon_{2} \mathbf{C}\right),
$$

where

$$
\mathbf{A}=\left(\begin{array}{cccc}
1 & 1 & \cdots & 1 \\
1 & 1 & \cdots & 1 \\
\vdots & \vdots & \ddots & \vdots \\
1 & 1 & \cdots & 1
\end{array}\right)_{m \times m}
$$

$\mathbf{B}=\left(\begin{array}{cccc}0 & 1 & \cdots & m-1 \\ 1 & 2 & \cdots & m \\ \vdots & \vdots & \ddots & \vdots \\ m-1 & m & \cdots & 2(m-1)\end{array}\right)_{m \times m}$,

C

$$
=-\frac{1}{2}\left(\begin{array}{ccccc}
0 & 1 & 2^{2 H} & \cdots & (m-1)^{2 H} \\
1 & 0 & \vdots & \cdots & (m-2)^{2 H} \\
\vdots & \vdots & \vdots & \ddots & \vdots \\
(m-1)^{2 H} & (m-2)^{2 H} & \cdots & \cdots & 0
\end{array}\right)_{m \times m}
$$

$c_{0}=2\left(1-2^{2 H-2}\right), \varepsilon_{1}=H / n, \varepsilon_{2}=1 / c_{0} n^{2 H}$.

Moreover, for a positive $\delta_{0}$, if the normalized approximation error is

$$
e(n) \triangleq \frac{\|\mathbf{K}(n, m)-\widehat{\mathbf{K}}(n, m)\|_{F}}{\|\mathbf{K}(n, m)\|_{F}} \leq \delta_{0},
$$

where $\|\cdot\|_{F}$ is the Frobenius norm, then

$$
\begin{aligned}
& n_{\min }=(m-1) \sqrt{\frac{H|2 H-1|}{\delta_{0}}}, \\
& \text { or } \delta_{0}=\left|\frac{H(2 H-1)(m-1)^{2}}{n_{\min }^{2}}\right| .
\end{aligned}
$$

Proof. By virtue of Taylor theorem, we have

$$
\begin{aligned}
(n+k)^{2 H}= & n^{2 H}+2 H \cdot k \cdot n^{2 H-1}+\frac{2 H(2 H-1)}{2 !} \cdot k^{2} \\
& \cdot n^{2 H-2}+\cdots .
\end{aligned}
$$

For $0<H<1,2 H-2<0$, the terms such as $n^{2 H-2}, n^{2 H-3}, \ldots$ on the right-hand side of (32) are negligible for large enough $n$.

Notice that $k_{m m}$ and $k_{11}$ are the largest and smallest term in the autocovariance $\mathbf{K}(n, m)$, respectively; $\left|k_{i j}-\widehat{k}_{i j}\right|$ is strictly increasing in indexes $i$ and $j$. The normalized approximation error $e(n)$ is defined as (30); that is,

$$
\begin{aligned}
e(n) & =\sqrt{\frac{\sum_{i=1}^{i=m} \sum_{j=1}^{j=m}\left|k_{i j}-\widehat{k}_{i j}\right|^{2}}{\sum_{i=1}^{i=m} \sum_{j=1}^{j=m}\left|k_{i j}\right|^{2}}} \leq \sqrt{\frac{m^{2}\left|k_{m m}-\widehat{k}_{m m}\right|^{2}}{m^{2}\left|k_{11}\right|^{2}}} \leq \frac{\left|k_{m m}-\widehat{k}_{m m}\right|}{\left|k_{11}\right|} \\
& =\left|\frac{2(n+m-1)^{2 H}-(1 / 2)(2 n+2 m-2)^{2 H}-c_{0} n^{2 H}-c_{0} H n^{2 H-1}(2 m-2)}{c_{0} n^{2 H}}\right| \approx\left|\frac{H(2 H-1)(m-1)^{2}}{n^{2}}\right|
\end{aligned}
$$

where $c_{0}=2\left(1-2^{2 H-2}\right)$. Therefore, the normalized approximation error $e(n)$ decays in $O\left(1 / n^{2}\right)$.
Given $\delta_{0}>0$ is the upper bound of error $e(n)$, we can obtain the minimum value of $n$ as follows: 


$$
n_{\min }=(m-1) \sqrt{\frac{H|2 H-1|}{\delta_{0}}},
$$

and $\delta_{0}$ tends to zero as $n_{\min } \rightarrow \infty$

Thus, for large enough $n \geq n_{\min }$, the approximate matrix $\widehat{\mathbf{K}}(n, m)$ of autocovariance matrix $\mathbf{K}(n, m)$ can be shown as follows:

$$
\widehat{\mathbf{K}}(n, m)=\left(\widehat{k}_{i j}\right)_{m \times m}^{(n)},
$$

where

$$
\begin{aligned}
\widehat{k}_{i j}= & V_{H} \\
& \cdot\left(c_{0} n^{2 H}+c_{0} H(i+j-2) n^{2 H-1}-\frac{1}{2}|i-j|^{2 H}\right),
\end{aligned}
$$

and $i, j=1,2, \ldots, m$.

That is,

$$
\widehat{\mathbf{K}}(n, m)=c_{0} n^{2 H} V_{H} \cdot\left(\mathbf{A}+\varepsilon_{1} \mathbf{B}+\varepsilon_{2} \mathbf{C}\right), \quad n \geq n_{\min },
$$

which completes the proof.

Remark 11. The $m \times m$ matrix $\mathbf{A}$ has two different eigenvalues, $\lambda_{1}=0$ with index one and multiplicity $m-1$, and $\lambda_{2} \doteq \lambda_{m}=$ $m$ with index one and multiplicity one and the corresponding eigenvector $\varphi_{m}=(1 / \sqrt{m}, 1 / \sqrt{m}, \ldots, 1 / \sqrt{m})^{T}$. And, the minimal polynomial of $\mathbf{A}$ is $p(\lambda)=\lambda\left(\lambda-\lambda_{2}\right)$.

Proposition 12. Let $\mathbf{A}, \mathbf{B}, \mathbf{C}, \varepsilon_{1}, \varepsilon_{2}$ be the same as Theorem 10 and $\lambda_{2} \doteq \lambda_{m}=m$ is the unique nonzero eigenvalue of $\mathbf{A}$ associated with the eigenvector $\varphi_{m} .\left(\varepsilon_{1} \mathbf{B}+\varepsilon_{2} \mathbf{C}\right)$ is a small perturbation to matrix $\mathbf{A}$; then $\hat{\lambda}_{m}$ denote the perturbed eigenvalue of $\left(\mathbf{A}+\varepsilon_{1} \mathbf{B}+\varepsilon_{2} \mathbf{C}\right)$ as follows:

(i) when $0<H<1 / 2$,

$$
\widehat{\lambda}_{m}=m+\frac{H m(m-1)}{n}-\frac{1}{c_{0} n^{2 H} m} \sum_{i=1}^{m-1} i(m-i)^{2 H},
$$

(ii) when $1 / 2<H<1$,

$$
\begin{aligned}
\hat{\lambda}_{m}= & m+\frac{H m(m-1)}{n}+\frac{m H^{2}\left(m^{2}-1\right)}{12 n^{2}} \\
& -\frac{1}{c_{0} n^{2 H} m} \sum_{i=1}^{m-1} i(m-i)^{2 H} .
\end{aligned}
$$

Proof. In terms of Theorem 10,

$$
\mathbf{A}+\varepsilon_{1} \mathbf{B}+\varepsilon_{2} \mathbf{C}=\mathbf{A}+\varepsilon_{2}\left(\frac{\varepsilon_{1}}{\varepsilon_{2}} \mathbf{B}+\mathbf{C}\right)=\mathbf{A}+\varepsilon_{2} \mathbf{A}^{(1)},
$$

and then the perturbation of $\mathbf{A}$ is linear in $\varepsilon_{2}$ with $\mathbf{A}\left(\varepsilon_{2}\right)=$ $\mathbf{A}+\varepsilon_{2} \mathbf{A}^{(1)}$. By virtue of (19), the corresponding first-order perturbation in $\lambda_{m}$ is

$$
\begin{aligned}
\lambda_{m}^{(1)} & =\varphi_{m}^{T} \mathbf{A}^{(1)} \varphi_{m}=\frac{\varepsilon_{1}}{\varepsilon_{2}} \varphi_{m}^{T} \mathbf{B} \varphi_{m}+\varphi_{m}^{T} \mathbf{C} \varphi_{m} \\
& =\frac{\varepsilon_{1}}{\varepsilon_{2}} m(m-1)-\frac{1}{m} \sum_{i=1}^{m-1} i(m-i)^{2 H} ;
\end{aligned}
$$

that is,

$$
\varepsilon_{2} \lambda_{m}^{(1)}=\varepsilon_{1} m(m-1)-\frac{\varepsilon_{2}}{m} \sum_{i=1}^{m-1} i(m-i)^{2 H} .
$$

Notice that the component matrices $\mathbf{Z}_{10}$ and $\mathbf{Z}_{20}$ of $\mathbf{A}$ corresponding to $\lambda_{1}=0$ and $\lambda_{2} \doteq \lambda_{m}=m$, respectively, can be written as

$$
\mathbf{Z}_{10}
$$

$$
=\left(\begin{array}{ccccc}
\frac{m-1}{m} & \frac{-1}{m} & \cdots & \frac{-1}{m} & \frac{-1}{m} \\
\frac{-1}{m} & \frac{m-1}{m} & \cdots & \frac{-1}{m} & \frac{-1}{m} \\
\vdots & \vdots & \ddots & \vdots & \vdots \\
\frac{-1}{m} & \frac{-1}{m} & \cdots & \frac{m-1}{m} & \frac{-1}{m} \\
\frac{-1}{m} & \frac{-1}{m} & \cdots & \frac{-1}{m} & \frac{m-1}{m}
\end{array}\right)_{m \times m},,
$$

By (20) and (21), the first-order perturbation in the eigenvector $\varphi_{m}$ is given as follows:

$$
\varphi_{m}^{(1)}=\mathbf{E}_{2} \mathbf{A}^{(1)} \varphi_{m},
$$

where $\mathbf{E}_{2}=(1 / m) \mathbf{Z}_{10}$; then

$$
\varphi_{m}^{(1)}=\frac{1}{m} \mathbf{Z}_{10}\left(\frac{\varepsilon_{1}}{\varepsilon_{2}} \mathbf{B}+\mathbf{C}\right) \varphi_{m}
$$

By (22), the second-order perturbation coefficient can be expressed as

$$
\begin{aligned}
\lambda_{m}^{(2)} & =\varphi_{m}^{T} \mathbf{A}^{(1)} \varphi_{m}^{(1)} \\
& =\frac{1}{m} \varphi_{m}^{T}\left(\frac{\varepsilon_{1}}{\varepsilon_{2}} \mathbf{B}+\mathbf{C}\right) \mathbf{Z}_{10}\left(\frac{\varepsilon_{1}}{\varepsilon_{2}} \mathbf{B}+\mathbf{C}\right) \varphi_{m}
\end{aligned}
$$

that is,

$$
\varepsilon_{2}^{2} \lambda_{m}^{(2)}=\frac{1}{m} \varphi_{m}^{T}\left(\varepsilon_{1} \mathbf{B}+\varepsilon_{2} \mathbf{C}\right) \mathbf{Z}_{10}\left(\varepsilon_{1} \mathbf{B}+\varepsilon_{2} \mathbf{C}\right) \varphi_{m} .
$$

If $0<H<1 / 2, \varepsilon_{1} \varepsilon_{2}=o\left(\varepsilon_{2}^{2}\right)$ and $\varepsilon_{1}^{2}=o\left(\varepsilon_{2}^{2}\right)$, as $n \rightarrow \infty$; if $1 / 2<H<1, \varepsilon_{1} \varepsilon_{2}=o\left(\varepsilon_{1}^{2}\right)$ and $\varepsilon_{2}^{2}=o\left(\varepsilon_{1}^{2}\right)$, as $n \rightarrow \infty$. Therefore,

(1) if $0<H<1 / 2$,

$$
\begin{aligned}
\varepsilon_{2}^{2} \lambda_{m}^{(2)} \approx & \frac{\varepsilon_{2}^{2}}{m} \varphi_{m}^{T} \mathbf{C Z}_{10} \mathbf{C} \varphi_{m} \\
= & \frac{\varepsilon_{2}^{2}}{16 m^{2}} \sum_{i=0}^{m-1}\left(\sum_{j=0}^{m-1}|i-j|^{2 H}\right)^{2} \\
& -\frac{\varepsilon_{2}^{2}}{4 m^{3}}\left(\sum_{i=0}^{m-1} i(m-i)^{2 H}\right)^{2},
\end{aligned}
$$


(2) if $1 / 2<H<1$,

$$
\varepsilon_{2}^{2} \lambda_{m}^{(2)} \approx \frac{\varepsilon_{1}^{2}}{m} \varphi_{m}^{T} \mathbf{B} \mathbf{Z}_{10} \mathbf{B} \varphi_{m} \approx \frac{m\left(m^{2}-1\right) \varepsilon_{1}^{2}}{12} .
$$

The third and other higher order perturbation in $\lambda_{m}$ are sufficiently small for large enough $n$ and the quantity of (49) is also sufficiently small; then $\hat{\lambda}_{m}$ denote the perturbed $\lambda_{m}$ as follows:

(i) when $0<H<1 / 2$,

$$
\widehat{\lambda}_{m}=m+\varepsilon_{1} m(m-1)-\frac{\varepsilon_{2}}{m} \sum_{i=1}^{m-1} i(m-i)^{2 H},
$$

(ii) when $1 / 2<H<1$,

$$
\begin{aligned}
\hat{\lambda}_{m}= & m+\varepsilon_{1} m(m-1)+\frac{m\left(m^{2}-1\right) \varepsilon_{1}^{2}}{12} \\
& -\frac{\varepsilon_{2}}{m} \sum_{i=1}^{m-1} i(m-i)^{2 H} .
\end{aligned}
$$

Submitting $\varepsilon_{1}, \varepsilon_{2}$ into (51) and (52), the proof is completed.

Proposition 13. Assume the autocovariance matrix

$$
\mathbf{K}(n, m)=\mathbf{Q}(n, m) \mathbf{\Lambda}(n, m) \mathbf{Q}(n, m)
$$

where $\mathbf{Q}(n, m)$ is an orthogonal matrix and $\mathbf{\Lambda}(n, m)$ is a diagonal matrix; then $\mathrm{K}(n, m)$ can be approximated as $\widehat{\mathbf{K}}(n, m)=\mathbf{Q}(n, m) \widehat{\Lambda}(n, m) \mathbf{Q}(n, m)$ for $n>n_{\text {min }}$, where $\widehat{\mathbf{K}}(n, m)$ is characterized as (28) and $\widehat{\Lambda}(n, m)$ is a diagonal matrix corresponding to $\widehat{\mathbf{K}}(n, m)$.

Proof. Since $\widehat{\mathbf{K}}(n, m)$ approaches to $\mathbf{K}(n, m)$ for large enough $n>n_{\text {min }}$, we have

$$
\begin{aligned}
\|\mathbf{K}(n, m) \widehat{\mathbf{K}}(n, m)-\widehat{\mathbf{K}}(n, m) \mathbf{K}(n, m)\|_{F} \longrightarrow & 0, \\
& n \longrightarrow \infty .
\end{aligned}
$$

The remaining is obvious, so we omit it.

Proposition 14. Suppose that $\hat{\lambda}_{m}$ is given the same as Proposition 12; then $\widehat{\mathbf{K}}(n, m)$ has a unique time varying eigenvalue $\hat{\lambda}_{m}(n)$ in asymptotic means (i.e., for large enough $n \geq n_{\min }$ ) as follows:

$$
\begin{aligned}
& \text { (i') when } 0<H<1 / 2 \text {, } \\
& \hat{\lambda}_{m}(n)=V_{H} \cdot\left(c_{0} m n^{2 H}+c_{0} H m(m-1) n^{2 H-1}\right. \\
& \left.-\frac{1}{m} \sum_{i=1}^{m-1} i(m-i)^{2 H}\right)
\end{aligned}
$$

(ii') when $1 / 2<H<1$,

$$
\begin{aligned}
& \hat{\lambda}_{m}(n)=V_{H}\left(c_{0} m n^{2 H}+c_{0} H m(m-1) n^{2 H-1}\right. \\
& \left.+\frac{c_{0} m\left(m^{2}-1\right) H^{2} n^{2 H-2}}{12}-\frac{1}{m} \sum_{i=1}^{m-1} i(m-i)^{2 H}\right) .
\end{aligned}
$$

Proof. By virtue of Theorem 10 and Proposition 12, it is obvious that $\widehat{\lambda}_{m}(n)$ is the eigenvalue of $\widehat{\mathbf{K}}(n, m)$. Now we prove that it is unique time varying eigenvalue in asymptotic means (i.e., for large enough $n \geq n_{\min }$ ). Firstly, we study the effect of perturbation $\varepsilon_{2} \mathbf{A}^{(1)}$ on the eigenvalue $\lambda_{1}=0$ of A which is index one and multiplicity $m-1$. According to Lemma $8, \lambda_{1}$ can be split into $\lambda_{11}\left(\varepsilon_{2}\right), \lambda_{12}\left(\varepsilon_{2}\right), \ldots, \lambda_{1(m-1)}\left(\varepsilon_{2}\right)$ for sufficiently small $\varepsilon_{2}, \lambda_{1 p}(0)=\lambda_{1}=0(p=1,2, \ldots, m-1)$, and $a_{1 p}$ is an eigenvalue of $\mathbf{Z}_{10} \mathbf{A}^{(1)} \mathbf{Z}_{10}, \lambda_{1 p}\left(\varepsilon_{2}\right)=\lambda_{1}+a_{1 p} \varepsilon_{2}+$ $o\left(\left|\varepsilon_{2}\right|^{1+1 / l}\right)$, as $\left|\varepsilon_{2}\right| \rightarrow 0, l \leq m-1$.

Thus, we express the eigenvalues $\widehat{\lambda}_{p}(n)$ of $\widehat{\mathbf{K}}(n, m)$ as

$$
\begin{aligned}
\hat{\lambda}_{p}(n) & =c_{0} n^{2 H} V_{H} \cdot\left(\lambda_{1}+a_{1 p} \varepsilon_{2}+o\left(\left|\varepsilon_{2}\right|^{1+1 / l}\right)\right) \\
& =V_{H} \cdot a_{1 p}+c_{0} n^{2 H} V_{H} \cdot o\left(\left|\frac{1}{c_{0} n^{2 H}}\right|^{1+1 / l}\right),
\end{aligned}
$$

where $p=1,2, \ldots, m-1$.

By (40) and (43),

$$
\begin{aligned}
\mathbf{Z}_{10} \mathbf{A}^{(1)} \mathbf{Z}_{10} & =\mathbf{Z}_{10}\left(\frac{\varepsilon_{1}}{\varepsilon_{2}} \mathbf{B}+\mathbf{C}\right) \mathbf{Z}_{10}=\mathbf{Z}_{10} \mathbf{C Z}_{10} \\
\mathbf{Z}_{10} \mathbf{B Z} \mathbf{Z}_{10} & =\mathbf{0}
\end{aligned}
$$

Notice that the matrix $\mathbf{C}$ is a constant matrix independent of $n$; then $a_{1 p}$ is an eigenvalue of constant matrix $\mathbf{Z}_{10} \mathbf{C Z}_{10}$ and $V_{H} \cdot a_{1 p}$ is also independent of $n$. On the other hand, the rank of $\mathbf{Z}_{10}$ is $m-1$ while $\mathbf{C}$ is full rank; then

$$
\operatorname{rank}\left(\mathbf{Z}_{10} \mathbf{A}^{(1)} \mathbf{Z}_{10}\right)=m-1
$$

which means that there are $m-1$ eigenvalues of index one for Hermitian matrix $\mathbf{Z}_{10} \mathbf{A}^{(1)} \mathbf{Z}_{10}$. According to Lemma 9, there exists a number $b_{1 p}$ corresponding to $a_{1 p}$ such that

$$
\begin{aligned}
\hat{\lambda}_{p}(n)= & V_{H} \cdot a_{1 p}+\frac{V_{H}}{c_{0} n^{2 H}} b_{1 p}+c_{0} n^{2 H} V_{H} \\
& \cdot o\left(\left|\frac{1}{c_{0} n^{2 H}}\right|^{2+1 / l}\right),
\end{aligned}
$$

and $b_{1 p}$ is an eigenvalue of $\mathbf{Z}_{10} \mathbf{A}^{(1)} \mathbf{E}_{1} \mathbf{A}^{(1)} \mathbf{Z}_{10}$ with right eigenvector $X_{1}$, where $X_{1}, X_{1,1}, \ldots, X_{1, l-1} \in \operatorname{Ker}\left(\mathbf{A}-\lambda_{1} \mathbf{I}\right)$ and $a_{1 p} X_{1}=\mathbf{Z}_{10} \mathbf{A}^{(1)} \mathbf{Z}_{10} X_{1}$.

By (21), (40), (43), and Theorem 10, we have $\mathbf{E}_{1}=-(1 /$ $\left.m^{2}\right) \mathbf{A}$ and

$$
\operatorname{rank}\left(\mathbf{Z}_{10} \mathbf{A}^{(1)} \mathbf{E}_{1} \mathbf{A}^{(1)} \mathbf{Z}_{10}\right)=1
$$


Here, it is indifferent to designate the unique nonzero Therefore, we obtain eigenvalue of $\mathbf{Z}_{10} \mathbf{A}^{(1)} \mathbf{E}_{1} \mathbf{A}^{(1)} \mathbf{Z}_{10}$ as $b_{1(m-1)}$. It is obvious that $b_{1 p}=0($ for $p=1,2, \ldots, m-2)$.

$$
\hat{\lambda}_{p}(n)= \begin{cases}V_{H} \cdot a_{1 p}+c_{0} n^{2 H} V_{H} \cdot o\left(\left|\frac{1}{c_{0} n^{2 H}}\right|^{1+1 / l}\right), & p=1,2, \ldots, m-2 ; \\ V_{H} \cdot a_{1 p}+\frac{V_{H}}{c_{0} n^{2 H}} b_{1 p}+c_{0} n^{2 H} V_{H} \cdot o\left(\left|\frac{1}{c_{0} n^{2 H}}\right|^{2+1 / l}\right), & p=m-1 .\end{cases}
$$

For large enough $n>n_{\min }$, we have

$$
\begin{aligned}
& \hat{\lambda}_{p}(n) \\
& \quad \approx \begin{cases}V_{H} \cdot a_{1 p}, & p=1,2, \ldots, m-2 ; \\
V_{H} \cdot a_{1 p}+\frac{V_{H}}{c_{0} n^{2 H}} b_{1 p}, & p=m-1 .\end{cases}
\end{aligned}
$$

Since the eigenvalues $\widehat{\lambda}_{1}(n), \ldots, \widehat{\lambda}_{m-2}(n)$ are independent of time in the asymptotic sense, $\widehat{\lambda}_{m-1}(n)$ decreases with the increasing of $n$ and it can be considered as independent of time; then we simply denote $\widehat{\lambda}_{1}(n), \widehat{\lambda}_{2}(n), \ldots, \hat{\lambda}_{m-1}(n)$ in above formula by $\hat{\lambda}_{1}, \hat{\lambda}_{2}, \ldots, \hat{\lambda}_{m-1}$, and the diagonal matrix $\widehat{\boldsymbol{\Lambda}}(n, m)$ associated with $\widehat{\mathbf{K}}(n, m)$ is expressed as follows:

$$
\widehat{\Lambda}(n, m)=\operatorname{diag}\left[\widehat{\lambda}_{1}, \widehat{\lambda}_{2}, \ldots, \widehat{\lambda}_{m-1}, \widehat{\lambda}_{m}(n)\right] .
$$

The proof is completed.

Remark 15. In view of Theorem 10 and Propositions 13 and 14, we can conclude that the unique eigenvalue of $\mathbf{K}(n, m)$ is time varying in asymptotic sense (i.e., for large enough $n \geq n_{\min }$ ).

Proposition 16. Let $X_{n, m}=Y_{n, m}-Y_{n-1, m}$ be the increment of dtsfBm $Y_{n, m}$ and let $\mathbf{R}(n, m)=E\left[X_{n, m} X_{n, m}^{T}\right]$ be the autocovariance matrix of $X_{n, m}$. Then $X_{n, m}$ is an asymptotic stationary process (i.e., for large enough $n \geq n_{\min }$ ) and the approximate autocovariance matrix $\widehat{\mathbf{K}}(n, m)$ of vector $Y_{n, m}$ is simultaneously diagonalizable with $\mathbf{R}(n, m)$ in asymptotic sense (i.e., for large enough $n \geq n_{\min }$ ).

Proof. It is easy to know that

$$
\begin{aligned}
& \mathbf{R}(n, m)=E\left[X_{n, m} X_{n, m}^{T}\right]=E\left[\left(Y_{n, m}-Y_{n-1, m}\right)\left(Y_{n, m}-Y_{n-1, m}\right)^{T}\right]=\left(r_{i j}\right)_{m \times m}^{(n)} \\
& =\left(\begin{array}{cccc}
E\left(S_{n}^{H}-S_{n-1}^{H}\right)^{2} & E\left(S_{n}^{H}-S_{n-1}^{H}\right)\left(S_{n+1}^{H}-S_{n}^{H}\right) & \cdots & E\left(S_{n}^{H}-S_{n-1}^{H}\right)\left(S_{n+m-1}^{H}-S_{n+m-2}^{H}\right) \\
E\left(S_{n+1}^{H}-S_{n}^{H}\right)\left(S_{n}^{H}-S_{n-1}^{H}\right) & E\left(S_{n+1}^{H}-S_{n}^{H}\right)^{2} & \cdots & E\left(S_{n+1}^{H}-S_{n}^{H}\right)\left(S_{n+m-1}^{H}-S_{n+m-2}^{H}\right) \\
\vdots & \vdots & \ddots & \vdots \\
E\left(S_{n+m-1}^{H}-S_{n+m-2}^{H}\right)\left(S_{n}^{H}-S_{n-1}^{H}\right) & E\left(S_{n+m-1}^{H}-S_{n+m-2}^{H}\right)\left(S_{n+1}^{H}-S_{n}^{H}\right) & \cdots & E\left(S_{n+m-1}^{H}-S_{n+m-2}^{H}\right)^{2}
\end{array}\right)_{m \times m}
\end{aligned}
$$

Considering (6), we have

$$
\begin{aligned}
r_{i j} & =E\left(S_{n+i-1}^{H} S_{n+j-1}^{H}\right)+E\left(S_{n+i-2}^{H} S_{n+j-2}^{H}\right) \\
& -E\left(S_{n+i-1}^{H} S_{n+j-2}^{H}\right)-E\left(S_{n+i-2}^{H} S_{n+j-1}^{H}\right) \\
& =\frac{V_{H}}{2}\left(2(2 n+i+j-3)^{2 H}-(2 n+i+j-2)^{2 H}\right. \\
& -(2 n+i+j-4)^{2 H}-2|i-j|^{2 H}+|i-j+1|^{2 H} \\
& \left.+|i-j-1|^{2 H}\right),
\end{aligned}
$$

where $i, j=1,2, \ldots, m$. By (32), for large enough $n>n_{\min }$,

$$
\begin{aligned}
& \frac{V_{H}}{2}\left(2(2 n+i+j-3)^{2 H}-(2 n+i+j-2)^{2 H}\right. \\
& \left.-(2 n+i+j-4)^{2 H}\right) \longrightarrow 0 .
\end{aligned}
$$

According to Theorem 10, we obtain

$$
\mathbf{R}(n, m)=V_{H}(2 \mathbf{C}-\mathbf{D}),
$$

where $\mathbf{C}$ is the same as in Theorem 10 and 


$$
\mathbf{D}=-\frac{1}{2}\left(\begin{array}{ccccc}
2 & 2^{2 H} & 3^{2 H}+1 & \cdots & m^{2 H}+(m-2)^{2 H} \\
2^{2 H} & 2 & 2^{2 H} & \cdots & (m-1)^{2 H}+(m-3)^{2 H} \\
\vdots & \vdots & \vdots & \ddots & \vdots \\
(m-1)^{2 H}+(m-3)^{2 H} & \cdot & \cdot & \cdots & 2^{2 H} \\
m^{2 H}+(m-2)^{2 H} & . & \cdot & \cdots & 2
\end{array}\right)_{m \times m}^{.}
$$

Hence, we show that the autocovariance matrix $\mathbf{R}(n, m)$ is independent of large enough time index $n$; that is, $X_{n, m}$ is an asymptotic stationary process.

Denote $\mathbf{R}(m)=\mathbf{R}(n, m)$ for simplicity. Assume that the diagonalization of $\mathbf{R}(m)$ is as follows:

$$
\mathbf{R}(m)=\mathbf{Q} \mathbf{\Lambda}_{m} \mathbf{Q}^{T},
$$

where $\mathbf{Q}$ is a constant orthogonal matrix independent of $n$. For $n>n_{\min }$, it is easy to verify that

$$
\begin{aligned}
\|\mathbf{R}(m) \widehat{\mathbf{K}}(n, m)-\widehat{\mathbf{K}}(n, m) \mathbf{R}(m)\|_{F} \longrightarrow & 0, \\
& \text { as } n \longrightarrow \infty,
\end{aligned}
$$

which means that the matrices $\mathbf{R}(m)$ and $\widehat{\mathbf{K}}(n, m)$ can be simultaneously diagonalized in asymptotic sense.

Now, on the basis of the above discussion, we receive the second main result.

Theorem 17 (structure theorem). The $m \times m$ autocovariance matrix $\mathbf{K}(n, m)$ can be diagonalizable as $\mathbf{K}(n, m)=$ $\mathbf{Q}(n, m) \mathbf{\Lambda}(n, m) \mathbf{Q}^{T}(n, m)$ and be approximated as $\widehat{\mathbf{K}}(n, m)$,

$$
\begin{aligned}
\widehat{\mathbf{K}}(n, m) & =\mathbf{Q}(n, m) \widehat{\Lambda}(n, m) \mathbf{Q}^{T}(n, m) \\
& \approx \mathbf{Q} \widehat{\Lambda}(n, m) \mathbf{Q}^{T},
\end{aligned}
$$

where

$$
\begin{aligned}
& \mathbf{Q}(n, m) \approx \mathbf{Q}=\text { constant orthogonal matrix, } \\
& \widehat{\Lambda}(n, m)=\operatorname{diag}\left\{\lambda_{1}, \lambda_{2}, \ldots, \lambda_{m-1}, \lambda_{m}(n)\right\},
\end{aligned}
$$

\section{Simulation and Illustration}

In this section, we simulate the eigenvalues and eigenvectors of the actual autocovariance matrices $\mathbf{K}(n, m)$ with different $H$ and $m$ by numerical experiment. The relative errors of time varying eigenvalues are given. Moreover, we analyze certain interesting behaviors of the autocovariance matrices (the data of Tables 1-7 are obtained by Maple; for details see Tables 1-7).

In Section 3, we show that the actual autocovariance matrices can be approximated as $\widehat{\mathbf{K}}(n, m)$. In Tables 1 and 2 , we express the constant orthogonal matrices $\mathbf{Q}$ by decomposing actual $\mathbf{K}(n, m)\left(n=2000\left(n \gg n_{\min }\right) ; H=0.4,0.8\right.$; $m=2,3, \ldots, 9)$. At the same time, Tables 1 and 2 are useful datum to those who need to know $\widehat{\mathbf{K}}(n, m)$ without computation at any time. Table 3 contains all eigenvalues of $\mathbf{K}(n, m)$ for $n=2000, H=0.1,0.2, \ldots, 0.4,0.6, \ldots, 0.9$, and $m=2,3, \ldots, 9$. In fact, it has been illustrated by these tables that all eigenvalues except for the largest one are time invariant and depend only on $H$. Table 4 shows the time varying eigenvalues and corresponding eigenvectors of actual autocovariance matrices of dtsfBm with $n=2000$, $H=0.1,0.2,0.3,0.6,0.7,0.9$, and $m=2,3, \ldots, 9$. On the other hand, the largest time varying eigenvalues can be approximated in (55) or (56). Table 5 reveals the relative error (|(actual - approximate)/actual|) between the actual eigenvalues and approximate values $\hat{\lambda}_{m}(n)$ in (55) or (56). Given $\delta_{0}>0$ be the upper bound of error $e(n)$, we can obtain the minimum values of $n$, that is, $n_{\min }$ in (34). Tables 6 and 7 list all $n_{\min }$ for $H=0.1,0.2, \ldots, 0.4,0.6, \ldots, 0.9$, $m=2,3, \ldots, 9, \delta_{0}=10^{-3}$, and $\delta_{0}=10^{-2}$, respectively. In the following, we give some comments from Tables 1-7.

(I) In Tables 1 and 2, the eigenvectors corresponding to the largest time varying eigenvalues always have nearly equal entries which hardly vary for $H=0.4,0.8$. In fact, these are also true for $H=0.1,0.2, \ldots, 0.4,0.6, \ldots, 0.9$ by consulting Tables 1, 2, and 4. In signal processing, these eigenvectors associated with the largest time varying eigenvalues correspond to a low-pass filter and are the direction of the average information of the signal if we consider them as the impulse response of an Finite Impulse Response (FIR) filter.

(II) By Table 5, we know that the approximation $\hat{\lambda}_{m}(n)$ of the largest time varying eigenvalue in (55) or (56) are very valid. Tables 3 and 4 can also verify Proposition 14 by numerical datum.

(III) Tables 6 and 7 illustrate that the approximation of autocovariance matrices in Theorems 10 and 17 are very well for $n_{\text {min }} \geq 200$. When $H=0.5, \mathrm{sfBm}$ is then a standard Brownian motion, the value of $n_{\text {min }}$ is zero, and this implies that the two theorems above are true for all values of time index $n$.

\section{Conclusion}

This article analyzes the autocovariance matrix of discrete time subfractional Brownian motion. The approximation and structure theorems of the autocovariance matrix are given. the unique largest time varying eigenvalues in asymptotic 
TABLE 1: The eigenvalues and eigenvectors of actual autocovariance matrices of dtsfBm with $n=2000$ and $H=0.4$.

\begin{tabular}{|c|c|c|}
\hline$m$ & $\begin{array}{c}\text { Eigenvalue } \\
H=0.4 n=2000\end{array}$ & Eigenvector \\
\hline \multirow{2}{*}{2} & 0.5645 & {$\left[\begin{array}{ll}-0.7072 & 0.7070\end{array}\right]^{T}$} \\
\hline & 1114.9 & {$\left[\begin{array}{ll}0.7070 & 0.7072\end{array}\right]^{T}$} \\
\hline \multirow{3}{*}{3} & 0.4250 & {$\left[\begin{array}{lll}0.4084 & -0.8164 & 0.4083\end{array}\right]^{T}$} \\
\hline & 0.9828 & {$\left[\begin{array}{lll}-0.7072 & -0.0001 & 0.7070\end{array}\right]^{T}$} \\
\hline & 1672.2 & {$\left[\begin{array}{lll}0.5772 & 0.5774 & 0.5774\end{array}\right]^{T}$} \\
\hline \multirow{4}{*}{4} & 0.3849 & {$\left[\begin{array}{llll}-0.2790 & 0.6498 & -0.6498 & 0.2789\end{array}\right]_{T}^{T}$} \\
\hline & 0.5853 & {$\left[\begin{array}{llll}0.5001 & -0.4999 & -0.5000 & 0.5000\end{array}\right]_{T}^{T}$} \\
\hline & 1.5388 & {$\left[\begin{array}{llll}0.6498 & 0.2791 & -0.2788 & -0.6497\end{array}\right]^{T}$} \\
\hline & 2229.4 & {$\left[\begin{array}{llll}0.4998 & 0.5000 & 0.5001 & 0.5001\end{array}\right]^{T}$} \\
\hline \multirow{5}{*}{5} & 0.3689 & {$\left[\begin{array}{lllll}0.1996 & -0.5132 & 0.6273 & -0.5132 & 0.1996\end{array}\right]_{T}^{T}$} \\
\hline & 0.4726 & {$\left[\begin{array}{lllll}-0.3820 & 0.5951 & 0.0000 & -0.5951 & 0.3819\end{array}\right]_{T}^{T}$} \\
\hline & 0.7914 & {$\left[\begin{array}{lllll}-0.5102 & 0.1911 & 0.6374 & 0.1913 & -0.5101\end{array}\right]_{T}^{T}$} \\
\hline & 2.2211 & {$\left[\begin{array}{lllll}0.5951 & 0.3821 & 0.0002 & -0.3818 & -0.5950\end{array}\right]^{T}$} \\
\hline & 2786.6 & {$\left[\begin{array}{lllll}0.4469 & 0.4472 & 0.4473 & 0.4474 & 0.4473\end{array}\right]^{T}$} \\
\hline \multirow{6}{*}{6} & 0.3602 & {$\left[\begin{array}{llllll}-0.1555 & 0.4105 & -0.5543 & 0.5543 & -0.4105 & 0.1555\end{array}\right]_{T}^{T}$} \\
\hline & 0.4265 & {$\left[\begin{array}{llllll}-0.2945 & 0.5773 & -0.2828 & -0.2829 & 0.5773 & -0.2945\end{array}\right]^{T}$} \\
\hline & 0.5856 & {$\left[\begin{array}{llllll}-0.4174 & 0.3964 & 0.4107 & -0.4106 & -0.3965 & 0.4173\end{array}\right]^{T}$} \\
\hline & 1.0370 & {$\left[\begin{array}{llllll}0.4968 & 0.0069 & -0.5032 & -0.5033 & 0.0067 & 0.4967\end{array}\right]_{T}^{T}$} \\
\hline & 3.0231 & {$\left[\begin{array}{llllll}-0.5493 & -0.2945 & -0.1553 & 0.1549 & 0.4173 & 0.5492\end{array}\right]^{T}$} \\
\hline & 3343.8 & {$\left[\begin{array}{llllll}0.4079 & 0.4082 & 0.4083 & 0.4084 & 0.4084 & 0.4083\end{array}\right]^{T}$} \\
\hline \multirow{7}{*}{7} & 0.3553 & {$\left[\begin{array}{lllllll}0.1226 & -0.3361 & 0.4798 & -0.5325 & 0.4798 & -0.3361 & 0.1226\end{array}\right]_{T}^{T}$} \\
\hline & 0.4010 & {$\left[\begin{array}{lllllll}0.2406 & -0.5220 & 0.4118 & 0.0000 & -0.4119 & 0.5220 & -0.2407\end{array}\right]_{T}^{T}$} \\
\hline & 0.5014 & {$\left[\begin{array}{lllllll}0.3391 & -0.4776 & -0.1267 & 0.5308 & -0.1266 & -0.4777 & 0.3390\end{array}\right]_{T}^{T}$} \\
\hline & 0.7189 & {$\left[\begin{array}{lllllll}0.4251 & -0.2160 & -0.5222 & -0.0001 & 0.5222 & 0.2161 & -0.4250\end{array}\right]^{T}$} \\
\hline & 1.3191 & {$\left[\begin{array}{lllllll}0.4768 & 0.1267 & -0.3328 & -0.5401 & -0.3330 & 0.1265 & 0.4767\end{array}\right]_{T}^{T}$} \\
\hline & 3.9403 & {$\left[\begin{array}{lllllll}0.5114 & 0.4254 & 0.2404 & 0.0002 & -0.2400 & -0.4251 & -0.5113\end{array}\right]^{T}$} \\
\hline & 3900.9 & {$\left[\begin{array}{lllllll}0.3776 & 0.3778 & 0.3780 & 0.3781 & 0.3781 & 0.3781 & 0.3780\end{array}\right]^{T}$} \\
\hline \multirow{8}{*}{8} & 0.3521 & {$\left[\begin{array}{llllllll}-0.1019 & 0.2810 & -0.4151 & 0.4883 & -0.4883 & 0.4151 & -0.2809 & 0.1019\end{array}\right]_{T}^{T}$} \\
\hline & 0.3861 & {$\left[\begin{array}{llllllll}-0.1971 & 0.4646 & -0.4574 & 0.1898 & 0.1898 & -0.4574 & 0.4646 & -0.1970\end{array}\right]_{T}^{T}$} \\
\hline & 0.4547 & {$\left[\begin{array}{llllllll}0.2872 & -0.4872 & 0.0881 & 0.4152 & -0.4152 & -0.0882 & 0.4872 & -0.2872\end{array}\right]_{T}^{T}$} \\
\hline & 0.5895 & {$\left[\begin{array}{llllllll}0.3585 & -0.3458 & -0.3611 & 0.3486 & 0.3486 & -0.3610 & -0.3459 & 0.3584\end{array}\right]_{T}^{T}$} \\
\hline & 0.8703 & {$\left[\begin{array}{llllllll}0.4208 & -0.0795 & -0.4875 & -0.2809 & 0.2808 & 0.4876 & 0.0796 & -0.4207\end{array}\right]_{T}^{T}$} \\
\hline & 1.6363 & {$\left[\begin{array}{llllllll}0.4559 & 0.1989 & -0.1878 & -0.4662 & -0.4662 & -0.1881 & 0.1987 & 0.4558\end{array}\right]_{T}^{T}$} \\
\hline & 4.9689 & {$\left[\begin{array}{llllllll}0.4797 & 0.4213 & 0.2870 & 0.1018 & -0.1013 & -0.2865 & -0.4210 & -0.4796\end{array}\right]^{T}$} \\
\hline & 4458.1 & {$\left[\begin{array}{llllllll}0.3531 & 0.3533 & 0.3535 & 0.3537 & 0.3537 & 0.3537 & 0.3537 & 0.3536\end{array}\right]^{T}$} \\
\hline \multirow{9}{*}{9} & 0.3499 & {$\left[\begin{array}{lllllllll}0.0848 & -0.2386 & 0.3610 & -0.4420 & 0.4694 & -0.4420 & 0.3610 & -0.2386 & 0.0848\end{array}\right]_{T}^{T}$} \\
\hline & 0.3760 & {$\left[\begin{array}{lllllllll}-0.1681 & 0.4117 & -0.4609 & 0.2997 & 0.0000 & -0.2998 & 0.4608 & -0.4117 & 0.1681\end{array}\right]^{T}$} \\
\hline & 0.4271 & {$\left[\begin{array}{lllllllll}-0.2422 & 0.4717 & -0.2277 & -0.2372 & -0.4686 & -0.2371 & -0.2277 & 0.4717 & -0.2422\end{array}\right]^{T}$} \\
\hline & 0.5181 & 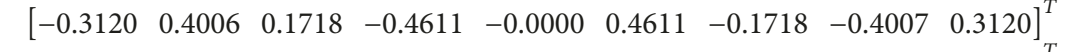 \\
\hline & 0.6890 & {$\left[\begin{array}{lllllllll}-0.3649 & 0.2252 & 0.4481 & -0.0744 & -0.4686 & -0.0745 & 0.4481 & 0.2253 & -0.3648\end{array}\right]^{T}$} \\
\hline & 1.0387 & {$\left[\begin{array}{lllllllll}-0.4115 & -0.0187 & 0.4011 & 0.4117 & 0.0001 & -0.4116 & -0.4012 & 0.0186 & 0.4114\end{array}\right]_{T}^{T}$} \\
\hline & 1.9873 & {$\left[\begin{array}{lllllllll}-0.4360 & -0.2425 & 0.0763 & 0.3628 & 0.4768 & 0.3629 & 0.0766 & -0.2423 & -0.4359\end{array}\right]^{T}$} \\
\hline & 6.1060 & 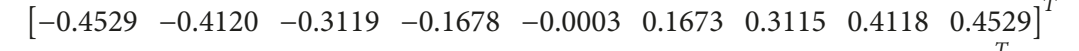 \\
\hline & 5015.3 & {$\left[\begin{array}{lllllllll}0.3329 & 0.3331 & 0.3333 & 0.3334 & 0.3335 & 0.3335 & 0.3335 & 0.3335 & 0.3334\end{array}\right]^{T}$} \\
\hline
\end{tabular}


TABLE 2: The eigenvalues and eigenvectors of actual autocovariance matrices of dtsfBm with $n=2000$ and $H=0.8$.

\begin{tabular}{|c|c|c|}
\hline$m$ & $\begin{array}{c}\text { Eigenvalue } \\
H=0.8 n=2000\end{array}$ & Eigenvector \\
\hline \multirow{2}{*}{2} & 0.5758 & {$\left[\begin{array}{ll}-0.7072 & 0.7070\end{array}\right]^{T}$} \\
\hline & $2.2055 \times 10^{5}$ & {$\left[\begin{array}{ll}0.7070 & 0.7072\end{array}\right]^{T}$} \\
\hline \multirow{3}{*}{3} & 0.1921 & {$\left[\begin{array}{lll}0.4083 & -0.8165 & 0.4082\end{array}\right]_{T}^{T}$} \\
\hline & 1.7272 & {$\left[\begin{array}{lll}0.7073 & 0.0003 & -0.7070\end{array}\right]_{T}^{T}$} \\
\hline & $3.3096 \times 10^{5}$ & {$\left[\begin{array}{lll}0.5771 & 0.5774 & 0.5776\end{array}\right]^{T}$} \\
\hline \multirow{4}{*}{4} & 0.1519 & {$\left[\begin{array}{llll}-0.2444 & 0.6636 & -0.6635 & 0.2443\end{array}\right]_{T}^{T}$} \\
\hline & 0.3759 & {$\left[\begin{array}{llll}0.5001 & -0.4999 & -0.5001 & 0.4999\end{array}\right]_{T}^{T}$} \\
\hline & 3.7024 & {$\left[\begin{array}{llll}-0.6637 & -0.2446 & 0.2440 & 0.6634\end{array}\right]^{T}$} \\
\hline & $4.4145 \times 10^{5}$ & {$\left[\begin{array}{llll}0.4997 & 0.4999 & 0.5001 & 0.5003\end{array}\right]^{T}$} \\
\hline \multirow{5}{*}{5} & 0.1341 & {$\left[\begin{array}{lllll}-0.1821 & 0.5064 & -0.6487 & 0.5064 & -0.1820\end{array}\right]_{T}^{T}$} \\
\hline & 0.2428 & {$\left[\begin{array}{lllll}0.3406 & -0.6198 & -0.0001 & 0.6197 & -0.3404\end{array}\right]^{T}$} \\
\hline & 0.6586 & {$\left[\begin{array}{lllll}-0.5167 & 0.2085 & 0.6158 & 0.2089 & -0.5165\end{array}\right]^{T}$} \\
\hline & 6.6462 & {$\left[\begin{array}{lllll}0.6199 & 0.3408 & 0.0004 & -0.3402 & -0.6196\end{array}\right]^{T}$} \\
\hline & $5.5203 \times 10^{5}$ & {$\left[\begin{array}{lllll}0.4469 & 0.4470 & 0.4472 & 0.4474 & 0.4476\end{array}\right]^{T}$} \\
\hline \multirow{6}{*}{6} & 0.1267 & {$\left[\begin{array}{llllll}-0.1314 & 0.3991 & -0.5691 & 0.5688 & -0.3986 & 0.1311\end{array}\right]_{T}^{T}$} \\
\hline & 0.1864 & {$\left[\begin{array}{llllll}0.2702 & -0.5768 & 0.3065 & 0.3069 & -0.5771 & 0.2703\end{array}\right]^{1}$} \\
\hline & 0.3740 & {$\left[\begin{array}{llllll}-0.3807 & 0.4431 & 0.3985 & -0.3984 & -0.4431 & 0.3806\end{array}\right]^{T}$} \\
\hline & 1.0512 & {$\left[\begin{array}{llllll}-0.5103 & 0.0209 & 0.4891 & 0.4892 & 0.0212 & -0.5100\end{array}\right]^{T}$} \\
\hline & 10.6886 & {$\left[\begin{array}{llllll}-0.5814 & -0.3806 & -0.1329 & 0.1320 & 0.3800 & 0.5811\end{array}\right]^{T}$} \\
\hline & $6.6270 \times 10^{5}$ & {$\left[\begin{array}{llllll}0.4078 & 0.4080 & 0.4082 & 0.4083 & 0.4085 & 0.4087\end{array}\right]^{T}$} \\
\hline \multirow{7}{*}{7} & 0.1221 & {$\left[\begin{array}{lllllll}0.1076 & -0.3235 & 0.4869 & -0.5418 & 0.4868 & -0.3235 & 0.1075\end{array}\right]_{T}^{T}$} \\
\hline & 0.1626 & {$\left[\begin{array}{lllllll}-0.2056 & 0.5148 & -0.4383 & 0.0005 & 0.4381 & -0.5159 & 0.2062\end{array}\right]^{T}$} \\
\hline & 0.2609 & {$\left[\begin{array}{lllllll}-0.3154 & 0.4939 & 0.0938 & -0.5448 & 0.0947 & 0.4928 & -0.3149\end{array}\right]_{T}^{T}$} \\
\hline & 0.5490 & {$\left[\begin{array}{lllllll}-0.3964 & 0.2792 & 0.5147 & 0.0001 & -0.5147 & -0.2794 & 0.3963\end{array}\right]_{T}^{T}$} \\
\hline & 1.5658 & {$\left[\begin{array}{lllllll}-0.4964 & -0.0957 & 0.3334 & 0.5165 & 0.3337 & -0.0953 & -0.4961\end{array}\right]^{T}$} \\
\hline & 15.9484 & {$\left[\begin{array}{lllllll}0.5483 & 0.3958 & 0.2080 & 0.0005 & -0.2071 & -0.3952 & -0.5481\end{array}\right]^{T}$} \\
\hline & $7.7346 \times 10^{5}$ & {$\left[\begin{array}{lllllll}0.3775 & 0.3777 & 0.3778 & 0.3780 & 0.3781 & 0.3783 & 0.3784\end{array}\right]^{T}$} \\
\hline \multirow{8}{*}{8} & 0.1194 & {$\left[\begin{array}{llllllll}0.0844 & -0.2656 & 0.4167 & -0.4968 & 0.4976 & -0.4187 & 0.2677 & -0.0853\end{array}\right]_{T}^{T}$} \\
\hline & 0.1477 & {$\left[\begin{array}{llllllll}0.1737 & -0.4523 & 0.4767 & -0.1990 & -0.1960 & 0.4751 & -0.4519 & 0.1736\end{array}\right]_{T}^{T}$} \\
\hline & 0.2135 & {$\left[\begin{array}{llllllll}0.2501 & -0.4972 & 0.1294 & 0.4175 & -0.4174 & -0.1290 & 0.4961 & -0.2494\end{array}\right]_{T}^{T}$} \\
\hline & 0.3574 & {$\left[\begin{array}{llllllll}0.3381 & -0.3768 & -0.3290 & 0.3675 & 0.3681 & -0.3289 & -0.3773 & 0.3382\end{array}\right]_{T}^{T}$} \\
\hline & 0.7703 & {$\left[\begin{array}{llllllll}0.4005 & -0.1515 & -0.4956 & -0.2668 & 0.2665 & 0.4955 & 0.1518 & -0.4003\end{array}\right]^{T}$} \\
\hline & 2.2138 & {$\left[\begin{array}{llllllll}0.4802 & 0.1690 & -0.2006 & -0.4480 & -0.4481 & -0.2010 & 0.1685 & 0.4800\end{array}\right]_{T}^{T}$} \\
\hline & 22.5349 & {$\left[\begin{array}{llllllll}-0.5198 & -0.3994 & -0.2519 & -0.0864 & 0.0855 & 0.2511 & 0.3988 & 0.5196\end{array}\right]^{T}$} \\
\hline & $8.8429 \times 10^{5}$ & {$\left[\begin{array}{llllllll}0.3531 & 0.3532 & 0.3533 & 0.3535 & 0.3536 & 0.3537 & 0.3539 & 0.3540\end{array}\right]^{T}$} \\
\hline \multirow{9}{*}{9} & 0.1174 & {$\left[\begin{array}{lllllllll}0.0723 & -0.2244 & 0.3590 & -0.4451 & 0.4773 & -0.4477 & 0.3630 & -0.2280 & 0.0736\end{array}\right]^{T}$} \\
\hline & 0.1392 & 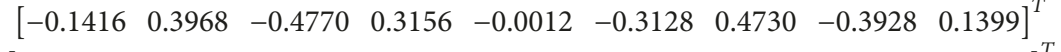 \\
\hline & 0.1845 & {$\left[\begin{array}{lllllllll}-0.2153 & 0.4678 & -0.2600 & -0.2303 & 0.4753 & -0.2291 & -0.2620 & 0.4696 & -0.2159\end{array}\right]^{T}$} \\
\hline & 0.2779 & 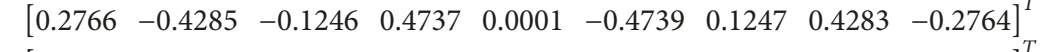 \\
\hline & 0.4767 & 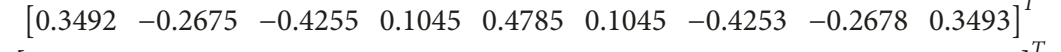 \\
\hline & 1.0414 & {$\left[\begin{array}{lllllllll}0.3986 & -0.0561 & -0.4263 & -0.3955 & -0.0001 & 0.3953 & 0.4263 & 0.0562 & -0.3982\end{array}\right]_{T}^{T}$} \\
\hline & 3.0064 & 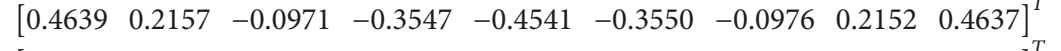 \\
\hline & 30.5512 & 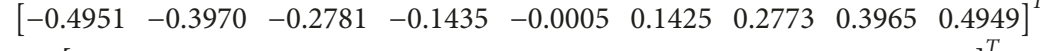 \\
\hline & $9.9522 \times 10^{5}$ & {$\left[\begin{array}{lllllllll}0.3328 & 0.3329 & 0.3331 & 0.3332 & 0.3333 & 0.3335 & 0.3336 & 0.3337 & 0.3338\end{array}\right]^{T}$} \\
\hline
\end{tabular}


TABLE 3: The eigenvalues for $n=2000$ and $H=0.1,0.2, \ldots, 0.4,0.6, \ldots, 0.9$.

\begin{tabular}{|c|c|c|c|c|c|c|c|c|}
\hline Eigenvalues & $H=0.1$ & 0.2 & 0.3 & 0.4 & 0.6 & 0.7 & 0.8 & 0.9 \\
\hline \multirow{2}{*}{$m=2$} & 1.7622 & 0.9587 & 0.6917 & 0.5645 & 0.4767 & 0.4949 & 0.5758 & 0.7896 \\
\hline & 44.2 & 106.5 & 328.0 & 1114.9 & 14864.7 & 56663.7 & $2.2055 \times 10^{5}$ & $8.7463 \times 10^{5}$ \\
\hline \multirow{3}{*}{$m=3$} & 1.6747 & 0.8565 & 0.5727 & 0.4250 & 0.2708 & 0.2257 & 0.1921 & 0.1675 \\
\hline & 2.0243 & 1.2651 & 1.0483 & 0.9828 & 1.0946 & 1.3023 & 1.7272 & 2.6539 \\
\hline & 65.2 & 159.1 & 491.5 & 1672.2 & 22303.1 & 85024.5 & $3.3096 \times 10^{5}$ & $1.3125 \times 10^{6}$ \\
\hline \multirow{4}{*}{$m=4$} & 1.6388 & 0.8185 & 0.5334 & 0.3849 & 0.2303 & 0.1854 & 0.1519 & 0.1269 \\
\hline & 1.8072 & 1.0002 & 0.7255 & 0.5853 & 0.4432 & 0.4036 & 0.3759 & 0.3579 \\
\hline & 2.3187 & 1.6280 & 1.4955 & 1.5388 & 2.0259 & 2.6015 & 3.7024 & 6.0461 \\
\hline & 86.2 & 211.6 & 654.9 & 2229.4 & 29745.5 & $1.1340 \times 10^{5}$ & $4.4145 \times 10^{5}$ & $1.7508 \times 10^{6}$ \\
\hline \multirow{5}{*}{$m=5$} & 1.6253 & 0.8040 & 0.5181 & 0.3689 & 0.2134 & 0.1680 & 0.1341 & 0.1086 \\
\hline & 1.7163 & 0.9008 & 0.6189 & 0.4726 & 0.3202 & 0.2756 & 0.2428 & 0.2170 \\
\hline & 1.9592 & 1.1711 & 0.9143 & 0.7914 & 0.6848 & 0.6647 & 0.6586 & 0.6634 \\
\hline & 2.6332 & 2.0335 & 2.0185 & 2.2211 & 3.2863 & 4.4486 & 6.6462 & 11.3163 \\
\hline & 107.0 & 263.9 & 818.1 & 2786.6 & 37191.8 & $1.4180 \times 10^{5}$ & $5.5203 \times 10^{5}$ & $2.1895 \times 10^{6}$ \\
\hline \multirow{6}{*}{$m=6$} & 1.6169 & 0.7953 & 0.5093 & 0.3602 & 0.2052 & 0.1602 & 0.1267 & 0.1008 \\
\hline & 1.6795 & 0.8604 & 0.5756 & 0.4265 & 0.2691 & 0.2221 & 0.1864 & 0.1580 \\
\hline & 1.8090 & 1.0015 & 0.7263 & 0.5856 & 0.4428 & 0.4028 & 0.3740 & 0.3538 \\
\hline & 2.1224 & 1.3607 & 1.1312 & 1.0370 & 0.9950 & 1.0131 & 1.0512 & 1.1094 \\
\hline & 2.9630 & 2.4748 & 2.6099 & 3.0231 & 4.8897 & 6.8923 & 10.6886 & 18.7933 \\
\hline & 127.7 & 316.1 & 981.2 & 3343.8 & 44642.2 & $1.7022 \times 10^{5}$ & $6.6270 \times 10^{5}$ & $2.6286 \times 10^{6}$ \\
\hline \multirow{7}{*}{$m=7$} & 1.6124 & 0.7906 & 0.5044 & 0.3553 & 0.2004 & 0.1554 & 0.1221 & 0.0956 \\
\hline & 1.6559 & 0.8357 & 0.5502 & 0.4010 & 0.2442 & 0.1978 & 0.1626 & 0.1366 \\
\hline & 1.7459 & 0.9310 & 0.6488 & 0.5014 & 0.3448 & 0.2974 & 0.2609 & 0.2329 \\
\hline & 1.9101 & 1.1141 & 0.8495 & 0.7189 & 0.5959 & 0.5664 & 0.5490 & 0.5396 \\
\hline & 2.2934 & 1.5654 & 1.3727 & 1.3191 & 1.3754 & 1.4548 & 1.5658 & 1.7135 \\
\hline & 3.3053 & 2.9477 & 3.2645 & 3.9403 & 6.8485 & 9.9760 & 15.9484 & 28.7840 \\
\hline & 148.4 & 368.2 & 1144.2 & 3900.9 & 52096.5 & $1.9866 \times 10^{5}$ & $7.7346 \times 10^{5}$ & $3.0681 \times 10^{6}$ \\
\hline \multirow{8}{*}{$m=8$} & 1.6092 & 0.7873 & 0.5011 & 0.3521 & 0.1974 & 0.1526 & 0.1194 & 0.0931 \\
\hline & 1.6426 & 0.8216 & 0.5356 & 0.3861 & 0.2290 & 0.1828 & 0.1477 & 0.1240 \\
\hline & 1.7051 & 0.8874 & 0.6034 & 0.4547 & 0.2972 & 0.2497 & 0.2135 & 0.1825 \\
\hline & 1.8193 & 1.0105 & 0.7332 & 0.5895 & 0.4381 & 0.3924 & 0.3574 & 0.3303 \\
\hline & 2.0169 & 1.2358 & 0.9860 & 0.8703 & 0.7792 & 0.7677 & 0.7703 & 0.7820 \\
\hline & 2.4707 & 1.7834 & 1.6368 & 1.6363 & 1.8276 & 1.9952 & 2.2138 & 2.4955 \\
\hline & 3.6584 & 3.4491 & 3.9781 & 4.9689 & 9.1732 & 13.7385 & 22.5349 & 41.5840 \\
\hline & 169.0 & 420.3 & 1307.1 & 4458.1 & 59554.7 & $2.2712 \times 10^{5}$ & $8.8429 \times 10^{5}$ & $3.5079 \times 10^{6}$ \\
\hline \multirow{9}{*}{$m=9$} & 1.6328 & 0.7852 & 0.4990 & 0.3499 & 0.1953 & 0.1506 & 0.1174 & 0.0919 \\
\hline & 1.6071 & 0.8116 & 0.5255 & 0.3760 & 0.2196 & 0.1737 & 0.1392 & 0.1151 \\
\hline & 1.6815 & 0.8621 & 0.5768 & 0.4271 & 0.2684 & 0.2208 & 0.1845 & 0.1531 \\
\hline & 1.7604 & 0.9465 & 0.6650 & 0.5181 & 0.3620 & 0.3147 & 0.2779 & 0.2508 \\
\hline & 1.8974 & 1.0967 & 0.8265 & 0.6890 & 0.5482 & 0.5072 & 0.4767 & 0.4532 \\
\hline & 2.1281 & 1.3653 & 1.1344 & 1.0387 & 0.9931 & 1.0083 & 1.0414 & 1.0888 \\
\hline & 2.6532 & 2.0132 & 1.9220 & 1.9873 & 2.3536 & 2.6395 & 3.0064 & 3.4756 \\
\hline & 4.0210 & 3.9767 & 4.7478 & 6.1060 & 11.8735 & 18.2155 & 30.5512 & 57.4685 \\
\hline & 189.5 & 472.2 & 1469.9 & 5015.3 & 67016.9 & $2.5559 \times 10^{5}$ & $9.9522 \times 10^{5}$ & $3.9482 \times 10^{6}$ \\
\hline
\end{tabular}


TABLE 4: The time varying eigenvalues and corresponding eigenvectors of actual autocovariance matrices of dtsfBm with $n=2000, H=$ $0.1,0.2,0.3,0.6,0.7,0.9$, and $m=2,3, \ldots, 9$.

\begin{tabular}{|c|c|c|c|}
\hline$m$ & $H$ & $\begin{array}{c}\text { Time varying } \\
\text { eigenvalue }\end{array}$ & Eigenvector \\
\hline \multirow{6}{*}{2} & 0.1 & 44.2 & {$\left[\begin{array}{ll}0.70709 & 0.70713\end{array}\right]^{T}$} \\
\hline & 0.2 & 106.5 & {$\left[\begin{array}{ll}0.70707 & 0.70714\end{array}\right]^{T}$} \\
\hline & 0.3 & 328.0 & {$\left[\begin{array}{ll}0.70705 & 0.70716\end{array}\right]^{T}$} \\
\hline & 0.6 & 14864.9 & {$\left[\begin{array}{ll}0.70700 & 0.70721\end{array}\right]^{T}$} \\
\hline & 0.7 & 56663.9 & {$\left[\begin{array}{ll}0.70698 & 0.70723\end{array}\right]^{T}$} \\
\hline & 0.9 & $8.7463 \times 10^{5}$ & {$\left[\begin{array}{ll}0.70695 & 0.70727\end{array}\right]^{T}$} \\
\hline \multirow{6}{*}{3} & 0.1 & 65.2 & {$\left[\begin{array}{lll}0.57652 & 0.57894 & 0.57659\end{array}\right]^{T}$} \\
\hline & 0.2 & 159.1 & {$\left[\begin{array}{lll}0.57692 & 0.57809 & 0.57704\end{array}\right]^{T}$} \\
\hline & 0.3 & 491.5 & {$\left[\begin{array}{lll}0.57712 & 0.57763 & .57730\end{array}\right]^{T}$} \\
\hline & 0.6 & 22303.1 & {$\left[\begin{array}{lll}0.57717 & 0.57736 & 0.57752\end{array}\right]^{T}$} \\
\hline & 0.7 & 85024.5 & {$\left[\begin{array}{lll}0.57715 & 0.57735 & 0.57755\end{array}\right]^{T}$} \\
\hline & 0.9 & $1.3125 \times 10^{6}$ & {$\left[\begin{array}{lll}0.57709 & 0.57735 & 0.57761\end{array}\right]^{T}$} \\
\hline \multirow{6}{*}{4} & 0.1 & 86.2 & {$\left[\begin{array}{llll}0.49867 & 0.50127 & 0.50130 & 0.49876\end{array}\right]^{T}$} \\
\hline & 0.2 & 211.6 & {$\left[\begin{array}{llll}0.49929 & 0.50060 & 0.50065 & 0.49945\end{array}\right]^{T}$} \\
\hline & 0.3 & 654.9 & {$\left[\begin{array}{llll}0.49964 & 0.50021 & 0.50028 & 0.49987\end{array}\right]^{T}$} \\
\hline & 0.6 & 29745.5 & {$\left[\begin{array}{llll}0.49976 & 0.49994 & 0.50009 & 0.50021\end{array}\right]^{T}$} \\
\hline & 0.7 & $1.1340 \times 10^{5}$ & {$\left[\begin{array}{llll}0.49973 & 0.49992 & 0.50009 & 0.50026\end{array}\right]^{T}$} \\
\hline & 0.9 & $1.7508 \times 10^{6}$ & {$\left[\begin{array}{llll}0.49966 & 0.49989 & 0.50011 & 0.50034\end{array}\right]^{T}$} \\
\hline \multirow{6}{*}{5} & 0.1 & 107.0 & {$\left[\begin{array}{llll}0.44558 & 0.44800 & 0.44875 & 0.448050 .44568\end{array}\right]^{T}$} \\
\hline & 0.2 & 263.9 & {$\left[\begin{array}{lllll}0.44632 & 0.44757 & 0.44800 & 0.44767 & 0.44650\end{array}\right]^{T}$} \\
\hline & 0.3 & 818.1 & {$\left[\begin{array}{lllll}0.44675 & 0.44731 & 0.44754 & 0.44745 & 0.44702\end{array}\right]^{T}$} \\
\hline & 0.6 & 37191.8 & {$\left[\begin{array}{lllll}0.44693 & 0.44709 & 0.44723 & 0.44736 & 0.44747\end{array}\right]^{T}$} \\
\hline & 0.7 & $1.4180 \times 10^{5}$ & {$\left[\begin{array}{lllll}0.44689 & 0.44706 & 0.44722 & 0.44737 & 0.44752\end{array}\right]^{T}$} \\
\hline & 0.9 & $2.1895 \times 10^{6}$ & {$\left[\begin{array}{lllll}0.44681 & 0.44701 & 0.44721 & 0.44742 & 0.44761\end{array}\right]^{T}$} \\
\hline \multirow{6}{*}{6} & 0.1 & 127.7 & $\left.\begin{array}{lllllll}0.40641 & 0.40861 & 0.40961 & 0.40964 & 0.40868 & 0.40653\end{array}\right]^{T}$ \\
\hline & 0.2 & 316.1 & {$\left[\begin{array}{llllll}0.40722 & 0.40838 & 0.40895 & 0.40899 & 0.40851 & 0.40743\end{array}\right]_{T}^{T}$} \\
\hline & 0.3 & 981.2 & {$\left[\begin{array}{llllll}0.40771 & 0.40824 & 0.40852 & 0.40859 & 0.40842 & 0.40801\end{array}\right]^{T}$} \\
\hline & 0.6 & 44642.2 & {$\left[\begin{array}{llllll}0.40792 & 0.40807 & 0.40820 & 0.40833 & 0.40844 & 0.40853\end{array}\right]^{T}$} \\
\hline & 0.7 & $1.7022 \times 10^{5}$ & {$\left[\begin{array}{llllll}0.40788 & 0.40804 & 0.40818 & 0.40833 & 0.40846 & 0.40860\end{array}\right]^{T}$} \\
\hline & 0.9 & $2.6286 \times 10^{6}$ & {$\left[\begin{array}{llllll}0.40779 & 0.40797 & 0.40816 & 0.40834 & 0.40852 & 0.40871\end{array}\right]^{T}$} \\
\hline \multirow{6}{*}{7} & 0.1 & 148.4 & {$\left[\begin{array}{lllllll}0.37600 & 0.37798 & 0.37906 & 0.37942 & 0.37910 & 0.37806 & 0.37612\end{array}\right]^{T}$} \\
\hline & 0.2 & 368.2 & {$\left[\begin{array}{lllllll}0.37683 & 0.37791 & 0.37852 & 0.37875 & 0.37860 & 0.37806 & 0.37707\end{array}\right]^{T}$} \\
\hline & 0.3 & 1144.2 & {$\left[\begin{array}{lllllll}0.37736 & 0.37786 & 0.37817 & 0.37831 & 0.37828 & 0.37808 & 0.37770\end{array}\right]^{T}$} \\
\hline & 0.6 & 52096.5 & {$\left[\begin{array}{lllllll}0.37760 & 0.37774 & 0.37787 & 0.37799 & 0.37809 & 0.37819 & 0.37828\end{array}\right]^{T}$} \\
\hline & 0.7 & $1.9865 \times 10^{5}$ & {$\left[\begin{array}{lllllll}0.37756 & 0.37770 & 0.37784 & 0.37797 & 0.37810 & 0.37823 & 0.37835\end{array}\right]^{T}$} \\
\hline & 0.9 & $3.0681 \times 10^{6}$ & {$\left[\begin{array}{lllllll}0.37745 & 0.37762 & 0.37780 & 0.37797 & 0.37814 & 0.37830 & 0.37847\end{array}\right]^{T}$} \\
\hline \multirow{6}{*}{8} & 0.1 & 169.0 & {$\left[\begin{array}{llllllll}0.35150 & 0.35329 & 0.35437 & 0.35489 & 0.35491 & 0.35443 & 0.35339 & 0.35163\end{array}\right]_{T}^{T}$} \\
\hline & 0.2 & 420.3 & {$\left[\begin{array}{llllllll}0.35234 & 0.35333 & 0.35396 & 0.35428 & 0.35432 & 0.35407 & 0.35352 & 0.35260\end{array}\right]_{T}^{T}$} \\
\hline & 0.3 & 1307.1 & {$\left[\begin{array}{llllllll}0.35289 & 0.35336 & 0.35368 & 0.35386 & 0.35391 & 0.35384 & 0.35363 & 0.35326\end{array}\right]_{T}^{T}$} \\
\hline & 0.6 & 59554.7 & {$\left[\begin{array}{llllllll}0.35315 & 0.35328 & 0.35341 & 0.35352 & 0.35363 & 0.35373 & 0.35381 & 0.35389\end{array}\right]^{T}$} \\
\hline & 0.7 & $2.2711 \times 10^{5}$ & {$\left[\begin{array}{llllllll}0.35311 & 0.35324 & 0.35337 & 0.35350 & 0.35362 & 0.35374 & 0.35386 & 0.35397\end{array}\right]^{T}$} \\
\hline & 0.9 & $3.5079 \times 10^{6}$ & {$\left[\begin{array}{llllllll}0.35299 & 0.35316 & 0.35332 & 0.35348 & 0.35363 & 0.35379 & 0.35395 & 0.35411\end{array}\right]^{T}$} \\
\hline \multirow{6}{*}{9} & 0.1 & 189.5 & 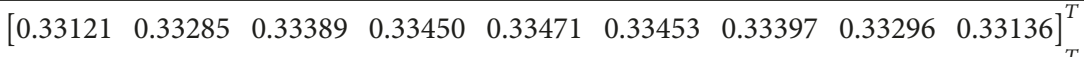 \\
\hline & 0.2 & 472.2 & $\left.\begin{array}{llllll}0.33397 & 0.33411 & 0.33403 & 0.33373 & 0.33318 & 0.33234\end{array}\right]^{T}$ \\
\hline & 0.3 & 1469.9 & $\left.\begin{array}{llllll}0.33359 & 0.33369 & 0.33369 & 0.33358 & 0.33336 & 0.33303\end{array}\right]^{T}$ \\
\hline & 0.6 & 67016.9 & $\left.\begin{array}{llllll}0.33325 & 0.33336 & 0.33345 & 0.33354 & 0.33362 & 0.33370\end{array}\right]^{T}$ \\
\hline & 0.7 & $2.5559 \times 10^{5}$ & $\left.\begin{array}{llllll}0.33323 & 0.33334 & 0.33346 & 0.33357 & 0.33368 & 0.33378\end{array}\right]_{T}^{T}$ \\
\hline & 0.9 & $3.9482 \times 10^{6}$ & {$\left[\begin{array}{lllllllll}0.33273 & 0.33288 & 0.33303 & 0.33319 & 0.33334 & 0.33348 & 0.33363 & 0.33378 & 0.33393\end{array}\right]^{T}$} \\
\hline
\end{tabular}


TABLE 5: The relative errors between the actual eigenvalues and the approximate values $\hat{\lambda}_{m}(n)$.

\begin{tabular}{lcccccc}
\hline Relative error & $H=0.4 n=200$ & $H=0.4 n=500$ & $H=0.4 n=2000$ & $H=0.8 n=200$ & $H=0.8 n=500$ & $H=0.8 n=2000$ \\
\hline$m=2$ & $1.9 \times 10^{-7}$ & $3.1 \times 10^{-8}$ & $1.8 \times 10^{-9}$ & $1.9 \times 10^{-5}$ & $3.0 \times 10^{-6}$ & $1.9 \times 10^{-7}$ \\
$m=3$ & $6.0 \times 10^{-7}$ & $6.0 \times 10^{-8}$ & $1.8 \times 10^{-9}$ & $5.3 \times 10^{-5}$ & $8.6 \times 10^{-6}$ & $5.4 \times 10^{-7}$ \\
$m=4$ & $1.6 \times 10^{-6}$ & $1.8 \times 10^{-7}$ & $2.2 \times 10^{-9}$ & $1.0 \times 10^{-4}$ & $1.7 \times 10^{-5}$ & $1.0 \times 10^{-6}$ \\
$m=5$ & $3.3 \times 10^{-6}$ & $4.0 \times 10^{-7}$ & $2.9 \times 10^{-9}$ & $1.7 \times 10^{-4}$ & $2.8 \times 10^{-5}$ & $1.1 \times 10^{-6}$ \\
$m=6$ & $5.7 \times 10^{-6}$ & $7.2 \times 10^{-7}$ & $1.3 \times 10^{-8}$ & $2.5 \times 10^{-4}$ & $4.1 \times 10^{-5}$ & $2.6 \times 10^{-6}$ \\
$m=7$ & $8.7 \times 10^{-6}$ & $1.1 \times 10^{-6}$ & $3.0 \times 10^{-8}$ & $3.5 \times 10^{-4}$ & $5.7 \times 10^{-5}$ & $3.6 \times 10^{-6}$ \\
$m=8$ & $1.2 \times 10^{-5}$ & $1.7 \times 10^{-6}$ & $5.1 \times 10^{-8}$ & $4.6 \times 10^{-4}$ & $7.5 \times 10^{-5}$ & $4.8 \times 10^{-6}$ \\
$m=9$ & $1.7 \times 10^{-5}$ & $2.3 \times 10^{-6}$ & $7.9 \times 10^{-8}$ & $5.9 \times 10^{-4}$ & $9.6 \times 10^{-5}$ & $6.1 \times 10^{-6}$ \\
\hline
\end{tabular}

TABLE 6: The values of $n_{\min }$ with $\delta_{0}=10^{-3}, H=0.1,0.2, \ldots, 0.9$, and $m=2,3, \ldots, 9$.

\begin{tabular}{|c|c|c|c|c|c|c|c|c|}
\hline$n_{\min }$ & $H=0.1$ & $H=0.2$ & $H=0.3$ & $H=0.4$ & $H=0.6$ & $H=0.7$ & $H=0.8$ & $H=0.9$ \\
\hline$m=2$ & 9 & 11 & 11 & 9 & 11 & 17 & 22 & 27 \\
\hline$m=3$ & 18 & 22 & 22 & 18 & 22 & 34 & 44 & 54 \\
\hline$m=4$ & 27 & 33 & 33 & 27 & 33 & 51 & 66 & 81 \\
\hline$m=5$ & 36 & 44 & 44 & 36 & 44 & 67 & 88 & 108 \\
\hline$m=6$ & 45 & 55 & 55 & 45 & 55 & 84 & 110 & 135 \\
\hline$m=7$ & 54 & 66 & 66 & 54 & 66 & 101 & 132 & 161 \\
\hline$m=8$ & 63 & 77 & 77 & 63 & 77 & 118 & 154 & 188 \\
\hline$m=9$ & 72 & 88 & 88 & 72 & 88 & 134 & 176 & 215 \\
\hline
\end{tabular}

TABLE 7: The values of $n_{\min }$ with $\delta_{0}=10^{-2}, H=0.1,0.2, \ldots, 0.9$, and $m=2,3, \ldots, 9$.

\begin{tabular}{lcccccccc}
\hline$n_{\min }$ & $H=0.1$ & $H=0.2$ & $H=0.3$ & $H=0.4$ & $H=0.6$ & $H=0.7$ & $H=0.8$ & $H=0.9$ \\
\hline$m=2$ & 3 & 4 & 4 & 3 & 4 & 6 & 7 \\
$m=3$ & 6 & 7 & 7 & 6 & 7 & 11 & 14 \\
$m=4$ & 9 & 11 & 11 & 9 & 11 & 16 & 21 \\
$m=5$ & 12 & 14 & 14 & 12 & 13 & 21 & 28 \\
$m=6$ & 15 & 18 & 18 & 15 & 18 & 27 & 35 \\
$m=7$ & 17 & 21 & 21 & 17 & 21 & 32 & 42 \\
$m=8$ & 20 & 25 & 25 & 20 & 25 & 38 & 43 \\
$m=9$ & 23 & 28 & 28 & 23 & 28 & 43 & 50 \\
\hline
\end{tabular}

sense are obtained. The eigenvectors corresponding to the largest time varying eigenvalues always have nearly equal entries which hardly vary for different $H \in(0,1)$. With numerical experiments, we inspect and verify the theoretical results. Though the increments of $\mathrm{sfBm}$ are nonstationary, we prove that the increments of dtsfBm are asymptotically stationary which fill with the blank space on nonstationary increments Gaussian process. It is believed that these results will be important to the further applications of $\mathrm{sfBm}$ in engineering, electron, network, and so forth. These are also our future works.

\section{Conflicts of Interest}

The author declares that there are no conflicts of interest regarding the publication of this paper.

\section{Acknowledgments}

This work is supported by NSF Grants 11471105 and 71561017 of China, NSF Grants 2016CFB526 of Hubei Province, and
Innovation Team of the Educational Department of Hubei Province T201412.

\section{References}

[1] F. Biagini, Y. Hu, B. Øksendal, and T. Zhang, Stochastic Calculus for Fractional Brownian Motion and Applications, Springer, London, UK, 2008.

[2] Y. S. Mishura, Stochastic Calculus for Fractional Brownian Motion and Related Processes, vol. 1929 of Lecture Notes in Mathematics, Springer, Berlin, Germany, 2008.

[3] T. Bojdecki, L. G. Gorostiza, and A. Talarczyk, "Sub-fractional Brownian motion and its relation to occupation times," Statistics \& Probability Letters, vol. 69, no. 4, pp. 405-419, 2004.

[4] T. Bojdecki, L. G. Gorostiza, and A. Talarczyk, "Fractional Brownian density process and its self-intersection local time of order k," Journal of Theoretical Probability, vol. 17, no. 3, pp. 717739, 2004.

[5] T. Bojdecki, L. G. Gorostiza, and A. Talarczyk, "Limit theorems for occupation time fluctuations of branching systems. I. Longrange dependence," Stochastic Processes and Their Applications, vol. 116, no. 1, pp. 1-18, 2006. 
[6] G. Shen and C. Chen, "Stochastic integration with respect to the subfractional Brownian motion with $H \in(0,12)$," Statistics \& Probability Letters, vol. 82, no. 2, pp. 240-251, 2012.

[7] C. Tudor, "Some properties of the sub-fractional Brownian motion," Stochastics, vol. 79, no. 5, pp. 431-448, 2007.

[8] C. Tudor, "Inner product spaces of integrands associated to subfractional Brownian motion," Statistics \& Probability Letters, vol. 78, no. 14, pp. 2201-2209, 2008.

[9] C. Tudor, "On the Wiener integral with respect to a subfractional Brownian motion on an interval," Journal of Mathematical Analysis and Applications, vol. 351, no. 1, pp. 456-468, 2009.

[10] L. Yan, G. Shen, and K. He, "Itô's formula for a sub-fractional Brownian motion," Communications on Stochastic Analysis, vol. 5, no. 1, pp. 135-159, 2011.

[11] G. Shen and L. Yan, "Estimators for the drift of subfractional Brownian motion," Communications in Statistics-Theory and Methods, vol. 43, no. 8, pp. 1601-1612, 2014.

[12] B. P. Rao, "Optimal estimation of a signal perturbed by a subfractional Brownian motion," Stochastic Analysis and Applications, vol. 35, no. 3, pp. 1-9, 2017.

[13] G. Shen and L. Yan, "An approximation of subfractional Brownian motion," Communications in Statistics-Theory and Methods, vol. 43, no. 9, pp. 1873-1886, 2014.

[14] J. Liu, D. Tang, and Y. Cang, "Variations and estimators for self-similarity parameter of sub-fractional Brownian motion via Malliavin calculus," Communications in Statistics-Theory and Methods, vol. 46, no. 7, pp. 3276-3289, 2017.

[15] J. Liu, L. Yan, Z. Peng, and D. Wang, "Remarks on confidence intervals for self-similarity parameter of a subfractional Brownian motion," Abstract and Applied Analysis, vol. 2012, Article ID 804942, 14 pages, 2012.

[16] T. J. Fisher, X. Sun, and C. M. Gallagher, "A new test for sphericity of the covariance matrix for high dimensional data," Journal of Multivariate Analysis, vol. 101, no. 10, pp. 2554-2570, 2010.

[17] A. Gupta and S. Joshi, "Some studies on the structure of covariance matrix of discrete-time fBm," IEEE Transactions on Signal Processing, vol. 56, no. 10, part 1, pp. 4635-4650, 2008.

[18] T. Ma, L. Jia, and Y. Su, "A new estimator of covariance matrix," Journal of Statistical Planning and Inference, vol. 142, no. 2, pp. 529-536, 2012.

[19] M. Packalen and T. S. Wirjanto, "Inference about clustering and parametric assumptions in covariance matrix estimation," Computational Statistics \& Data Analysis, vol. 56, no. 1, pp. 1-14, 2012.

[20] Y. Sheena and A. Takemura, "Admissible estimator of the eigenvalues of the variance-covariance matrix for multivariate normal distributions," Journal of Multivariate Analysis, vol. 102, no. 4, pp. 801-815, 2011.

[21] N. Su and R. Lund, "Multivariate versions of Bartlett's formula," Journal of Multivariate Analysis, vol. 105, pp. 18-31, 2012.

[22] T.-H. Sun, C.-S. Liu, and F.-C. Tien, "Invariant 2D object recognition using eigenvalues of covariance matrices, re-sampling and autocorrelation," Expert Systems with Applications, vol. 35, no. 4, pp. 1966-1977, 2008.

[23] W. B. Wu and M. Pourahmadi, "Banding sample autocovariance matrices of stationary processes," Statistica Sinica, vol. 19, no. 4, pp. 1755-1768, 2009.

[24] A. Ayache, S. Cohen, and J. L. Véhel, “The covariance structure of multifractional Brownian motion, with application to long range dependence," in Proceedings of the 25th IEEE International Conference on Acoustics, Speech, and Signal Processing (ICASSP '00), pp. 3810-3813, June 2000.
[25] E. Perrin, R. Harba, C. Berzin-Joseph, I. Iribarren, and A. Bonami, "nth-order fractional Brownian motion and fractional Gaussian noises," IEEE Transactions on Signal Processing, vol. 49, no. 5, pp. 1049-1059, 2001.

[26] E. Perrin, R. Harba, R. Jennane, and I. Iribarren, "Fast and exact synthesis for 1-D fractional Brownian motion and fractional Gaussian noises," IEEE Signal Processing Letters, vol. 9, no. 11, pp. 382-384, 2002.

[27] P. Lancaster and M. Tismenetsky, The Theory of Matrices, Academic Press, New York, NY, USA, 2nd edition, 1985. 


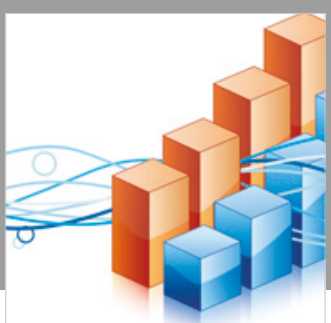

Advances in

Operations Research

\section{-n-m}
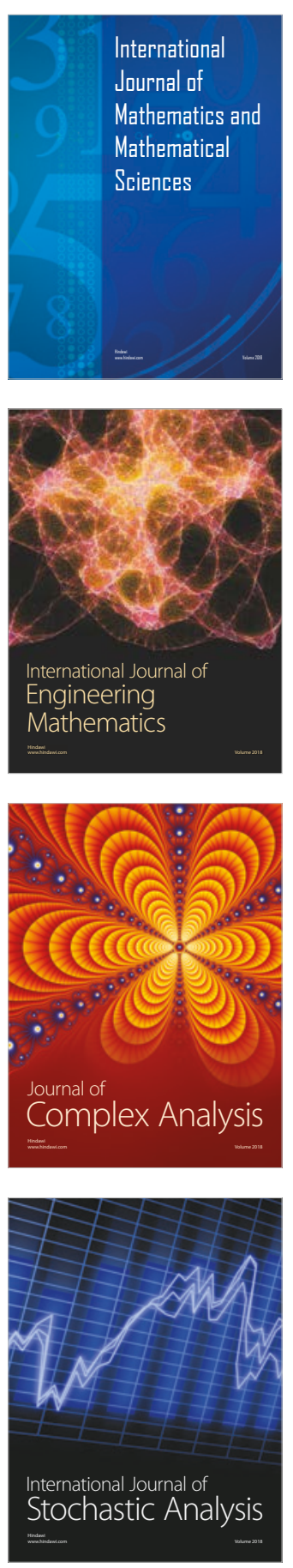
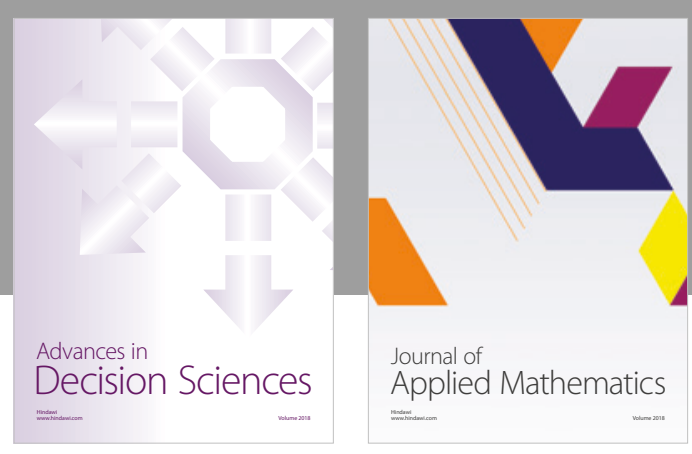

Journal of

Applied Mathematics
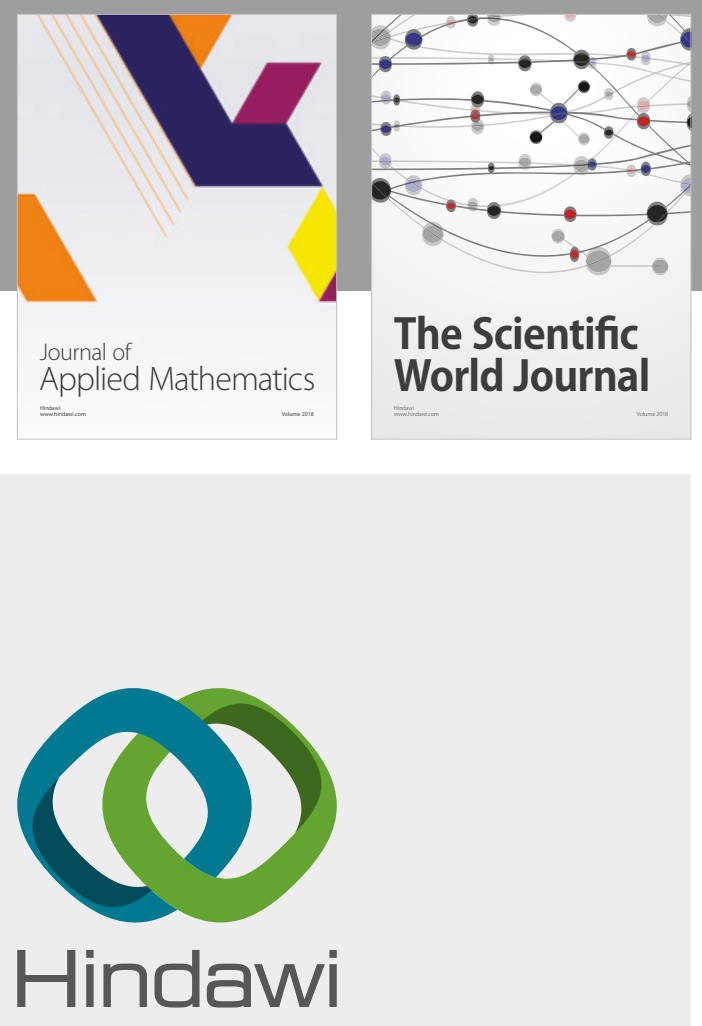

Submit your manuscripts at

www.hindawi.com

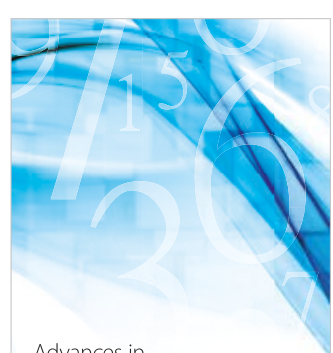

Advances in
Numerical Analysis
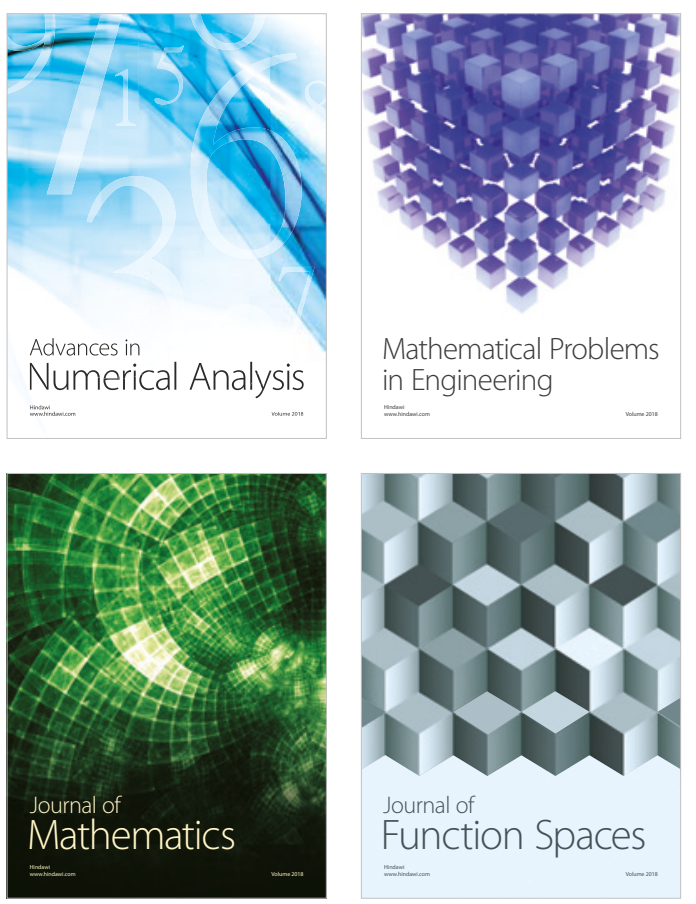

Mathematical Problems in Engineering

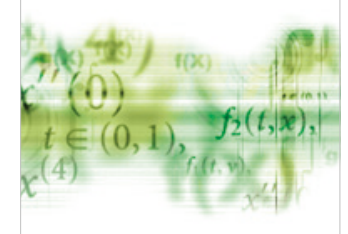

International Journal of

Differential Equations

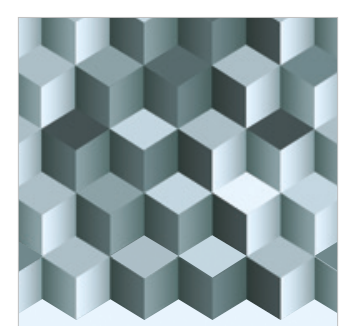

Journal of

Function Spaces

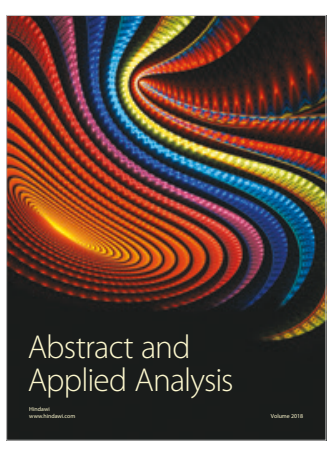

The Scientific

World Journal

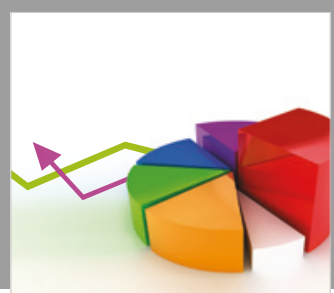

Journal of

Probability and Statistics
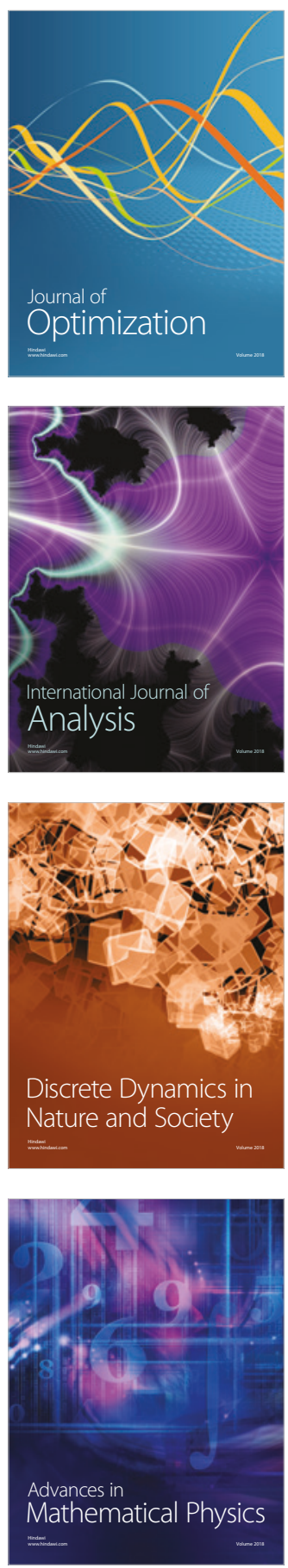